San Jose State University

SJSU ScholarWorks

Faculty Publications

Physics and Astronomy

$1-1-2012$

\title{
The ongoing assembly of a central cluster galaxy: phase-space substructures in the halo of M87
}

Aaron J. Romanowsky

San Jose State University, aaron.romanowsky@sjsu.edu

J. Strader

Harvard-Smithsonian Center for Astrophysics

J. P. Brodie

University of California Observatories

C. Mihos

Case Western Reserve University

L. R. Spitler

Swinburne University

See next page for additional authors

Follow this and additional works at: https://scholarworks.sjsu.edu/physics_astron_pub

Part of the Astrophysics and Astronomy Commons

\section{Recommended Citation}

Aaron J. Romanowsky, J. Strader, J. P. Brodie, C. Mihos, L. R. Spitler, D. A. Forbes, C. Foster, and J. A. Arnold. "The ongoing assembly of a central cluster galaxy: phase-space substructures in the halo of M87" Astrophysical Journal (2012). https://doi.org/10.1088/0004-637X/748/1/29

This Article is brought to you for free and open access by the Physics and Astronomy at SJSU ScholarWorks. It has been accepted for inclusion in Faculty Publications by an authorized administrator of SJSU ScholarWorks. For more information, please contact scholarworks@sjsu.edu. 


\section{Authors}

Aaron J. Romanowsky, J. Strader, J. P. Brodie, C. Mihos, L. R. Spitler, D. A. Forbes, C. Foster, and J. A. Arnold 


\title{
THE ONGOING ASSEMBLY OF A CENTRAL CLUSTER GALAXY: PHASE-SPACE SUBSTRUCTURES IN THE HALO OF M87
}

\author{
Aaron J. Romanowsky ${ }^{1}$, Jay Strader ${ }^{2}$, Jean P. Brodie ${ }^{1}$, J. Christopher Mihos ${ }^{3}$, Lee R. Spitler ${ }^{4}$, \\ Duncan A. Forbes ${ }^{4}$, CAROline Foster ${ }^{4,5}$, And Jacob A. Arnold ${ }^{1}$ \\ ${ }^{1}$ University of California Observatories, 1156 High Street, Santa Cruz, CA 95064, USA \\ ${ }^{2}$ Harvard-Smithsonian Center for Astrophysics, Cambridge, MA 02138, USA \\ ${ }^{3}$ Department of Astronomy, Case Western Reserve University, 10900 Euclid Ave., Cleveland, OH 44106, USA \\ ${ }^{4}$ Centre for Astrophysics and Supercomputing, Swinburne University, Hawthorn, VIC 3122, Australia \\ ${ }^{5}$ European Southern Observatory, Alonso de Córdova 3107, Vitacura, Casilla 19001, Santiago 19, Chile \\ Received 2011 July 20; accepted 2011 December 16; published 2012 March 2
}

\begin{abstract}
The halos of galaxies preserve unique records of their formation histories. We carry out the first combined observational and theoretical study of phase-space halo substructure in an early-type galaxy: M87, the central galaxy in the Virgo cluster. We analyze an unprecedented wide-field, high-precision photometric and spectroscopic data set for 488 globular clusters (GCs), which includes new, large-radius Subaru/Suprime-Cam and Keck/DEIMOS observations. We find signatures of two substructures in position-velocity phase space. One is a small, cold stream associated with a known stellar filament in the outer halo; the other is a large shell-like pattern in the inner halo that implies a massive, hitherto unrecognized accretion event. We perform extensive statistical tests and independent metallicity analyses to verify the presence and characterize the properties of these features, and to provide more general methodologies for future extragalactic studies of phase-space substructure. The cold outer stream is consistent with a dwarf galaxy accretion event, while for the inner shell there is tension between a low progenitor mass implied by the cold velocity dispersion, and a high mass from the large number of GCs, which might be resolved by a $\sim 0.5 L^{*} \mathrm{E} / \mathrm{S} 0$ progenitor. We also carry out proof-of-principle numerical simulations of the accretion of smaller galaxies in an M87-like gravitational potential. These produce analogous features to the observed substructures, which should have observable lifetimes of $\sim 1$ Gyr. The shell and stream GCs together support a scenario where the extended stellar envelope of M87 has been built up by a steady rain of material that continues until the present day. This phase-space method demonstrates unique potential for detailed tests of galaxy formation beyond the Local Group.
\end{abstract}

Key words: galaxies: elliptical and lenticular, $\mathrm{cD}$ - galaxies: formation - galaxies: halos - galaxies: individual (M87) - galaxies: kinematics and dynamics - galaxies: star clusters: general

Online-only material: color figures, machine-readable tables

\section{INTRODUCTION}

The ultimate record of the formational histories of galaxies is found in the six-dimensional phase space of stellar positions and velocities, supplemented by additional information about ages and elemental abundances (primarily iron-based metallicities). The halos of galaxies are natural hunting grounds for assembly clues in phase space because of the preservative effects of the long dynamical times, and since any material entering a galaxy naturally has to traverse its halo.

Indeed, it is a fundamental prediction of the current cosmological paradigm that the outer regions of galaxies should be constantly bombarded with smaller, infalling systems that persist as significant substructures while gradually becoming disrupted. This idea has been borne out qualitatively in recent years by observations of halo streams and shells in the nearby universe (e.g., Helmi et al. 1999; Ibata et al. 2001; Belokurov et al. 2006; Tal et al. 2009; Martínez-Delgado et al. 2010; Cooper et al. 2011).

Massive elliptical galaxies are touchstones for this issue, since their spheroidal nature and their residence in the densest areas of the universe suggest particularly vigorous accretion histories. Comparisons of these galaxies' stellar luminosity profiles at high and low redshifts now provide indirect evidence that their outer envelopes grow tremendously in size up to the present day (e.g., Buitrago et al. 2008; van der Wel et al. 2008; Damjanov et al. 2009; van Dokkum et al. 2010; Cassata et al. 2011), which may be naturally explained by accretion and mergers in a cosmological context (Khochfar \& Silk 2006; Naab et al. 2009; Hopkins et al. 2010; Oser et al. 2010, 2012).

The most extreme examples of ongoing assembly are expected to be the brightest cluster galaxies (BCGs), whose typical locations at the centers of clusters should involve very active merger histories (e.g., Ruszkowski \& Springel 2009). ${ }^{6}$ Current cosmologically based models find that around half of the stellar mass growth of a present-day BCG occurred over the past $5 \mathrm{Gyr}$ via gas-poor mergers (after an early formation epoch for the core regions; De Lucia \& Blaizot 2007).

This theoretical picture has received very mixed support from comparisons of BCG stellar masses and surface brightness distributions at different redshifts (Whiley et al. 2008; Bernardi 2009; Collins et al. 2009a; Valentinuzzi et al. 2010; Ascaso et al. 2011; Stott et al. 2011). Surveys for major mergers in action have found that these contribute significantly to recent BCG growth (McIntosh et al. 2008; Liu et al. 2009; see also Brough et al. 2011), but the contributions from minor mergers are unknown and the overall comparison to theory is unclear. Part of the difficulty is that even if accretion and mergers are important

\footnotetext{
6 Although the central galaxy is often not technically the brightest object in a cluster, we will follow the convention of calling this the BCG.
} 
growth processes, the frequency of observing an ongoing event can be fairly low. Thus, it would be invaluable to go beyond purely photometric observations and delve into the phase space of position, velocity, and metallicity for longer lived signatures (e.g., Johnston et al. 2008; Zemp et al. 2009).

Within the Local Group, detailed phase-space studies are being undertaken using individual stars (e.g., Koch et al. 2008; Gilbert et al. 2009; Starkenburg et al. 2009; Xue et al. 2011), but are currently impossible in more distant galaxies. Instead, bright stellar proxies may be used, such as planetary nebulae (PNe; Durrell et al. 2003; Merrett et al. 2003; Shih \& Méndez 2010; Cortesi et al. 2011) and globular clusters (GCs). GCs are thought to form along with field stars, and as collections of $\sim 10^{6}$ stars each, they are visible at much greater distances, allowing their individual positions, line-of-sight velocities, and chemical properties to be measured as far away as $\sim 50 \mathrm{Mpc}$ (Richtler et al. 2011).

The "chemodynamics" of GCs provided the watershed evidence for the accretion origin of the Milky Way's outer halo (Searle \& Zinn 1978), where it is now thought that disrupting satellite galaxies have deposited many accompanying GCs (e.g., Bellazzini et al. 2003; Gao et al. 2007; Geisler et al. 2007; Lee et al. 2007; Forbes \& Bridges 2010). A similar scenario is now evident in the nearby spiral M31, where some GCs are associated with halo substructures (Collins et al. 2009b; Mackey et al. 2010). In BCGs, the presence of GCs in enormous numbers (up to $5 \times 10^{4}$ per system) has long motivated suggestions that accretion is an important factor for these galaxies (Forte et al. 1982; Côté et al. 1998), although it is not clear how many of these GCs may have formed during in situ processes or mergers rather than being accreted.

Various GC- and PN-based studies of the halo kinematics of giant ellipticals and BCGs have turned up evidence for substructures that may imply active accretion events or mergers (Côté et al. 2003; Romanowsky et al. 2009; Schuberth et al. 2010; McNeil et al. 2010; Woodley \& Harris 2011). In other cases, accretion events are suggested by photometric substructures or by broad kinematical or chemical halo transitions (Tal et al. 2009; Proctor et al. 2009; Coccato et al. 2009, 2010; Janowiecki et al. 2010; Forbes et al. 2011; Arnold et al. 2011; Mouhcine et al. 2011). However, in no case has a halo feature been observed and modeled in enough detail to determine its origin and the implications for galaxy formation.

To go beyond the previous work on nearby BCGs by carrying out detailed phase-space studies of their halos, there are two critical observational requirements beside sheer instrumental throughput: wide-field coverage and spectroscopic resolution. The former permits blind searches for halo substructures and demands a field of view of at least $\sim 0.5^{\circ}$ in order to probe galactocentric radii out to $\sim 100 \mathrm{kpc}$ or more in galaxies at $\sim 15 \mathrm{Mpc}$ distances. The latter allows for a wide mass range of accreted galaxies to be probed; e.g., if a BCG has a characteristic velocity dispersion of $\sigma \sim 300 \mathrm{~km} \mathrm{~s}^{-1}$ and the precision of velocity measurements for halo tracers is $\Delta v \sim 50-100 \mathrm{~km} \mathrm{~s}^{-1}$, as in many past surveys, then the observations are sensitive to mass ratios down to $\sim(\Delta v / \sigma)^{2} \sim 1: 40-1: 10$ in dynamical mass. More minor mergers than these are certainly expected to be more frequent, and may comprise a dominant mode for halo assembly, so a velocity resolution of tens of $\mathrm{km} \mathrm{s}^{-1}(\Delta v / \sigma \lesssim 0.1)$ is preferable.

We present here the first wide-field, large-sample spectroscopic survey of a BCG with velocity resolution of $\Delta v / \sigma \sim$ 0.04. Our subject is M87, the central elliptical in Virgo, the near- est galaxy cluster at a distance of $\sim 16.5 \mathrm{Mpc}$. The GC system of M87 has seen decades of spectroscopic study (e.g., Huchra \& Brodie 1987; Cohen \& Ryzhov 1997; Hanes et al. 2001), but extending to radii of only $\sim 40 \mathrm{kpc}$ and with $\Delta v / \sigma \gtrsim 0.25$.

Until now, M87 could be characterized as a dynamically quiet galaxy, with only mild signs of interactions such as very faint stellar filaments in its far outer halo (Mihos et al. 2005; Janowiecki et al. 2010). Our new GC study reveals the kinematics of one of these faint streams, and unveils an unsuspected, enormous shell-like substructure in phase space.

Our paper proceeds as follows. Section 2 describes the observations and data reduction, and presents a basic overview of the halo substructures. Sections 3 and 4 analyze the characteristics and statistical significance of the large inner shell and outer stream, respectively. Theoretical analyses of the substructure origins and dynamics are explored in Section 5. Section 6 summarizes the findings and outstanding questions.

\section{OBSERVATIONS AND BASIC RESULTS}

We have revisited M87 in a new era high-precision wide-field survey of GCs. The main component of our data set is presented and discussed in detail in Strader et al. (2011, hereafter S+11). In brief, it is based on photometry from Canada-France-Hawaii Telescope (CFHT)/Megacam and Subaru/Suprime-Cam optical images, and spectroscopy from MMT/Hectospec, Keck/DEIMOS, and Keck/LRIS. New line-of-sight velocities were obtained for $451 \mathrm{GCs}$ over a series of campaigns from 2007 to 2010 , extending over a range in galactocentric radius of $\sim 1-35 \operatorname{arcmin}(\sim 5-170 \mathrm{kpc})$.

The first subset of the new data extended along a narrow track eastward of M87's center, in search of any kinematical transition between the BCG and the surrounding cluster. A peculiar fluctuation in the velocity dispersion profile was indeed found at a radius of $\sim 9 \operatorname{arcmin}(\sim 45 \mathrm{kpc})$, which was the first indication of an inner-halo shell-like feature as we will discuss later. Subsequent data (the large majority of the total sample) were obtained at generally random position angles around the galaxy.

In a parallel campaign presented here for the first time, we observed intensively the region around the outer stellar "stream A" (Mihos et al. 2005; Janowiecki et al. 2010; Rudick et al. 2010; Krick et al. 2011), at radii of $\sim 35-50$ arcmin ( $\sim 170-240 \mathrm{kpc})$. GC selection for spectroscopic follow-up was obtained from a variety of images as they became available: archival Subaru/Suprime-Cam (Miyazaki et al. 2002) BVI imaging, the Megacam gri imaging mentioned above (Harris 2009), and our own new Suprime-Cam imaging in $g i$. Given the incomplete spatial coverage of the first two imaging data sets, the third data set provides our default photometric source for the stream area. These images were taken on 2009 April 21, with intermittent transparency, $\sim 1^{\prime \prime}$ seeing, and exposure times of $300 \mathrm{~s}$ in each band.

Our spectroscopic follow-up of the stream GCs used Keck/ DEIMOS and observing and analysis techniques established in our previous work on GC kinematics (Romanowsky et al. 2009; Arnold et al. 2011; Foster et al. 2011; S+11). Four masks were observed during the nights 2009 March 23, 2010 March 11-13, and 2010 June 12, with generally good conditions and exposure times varying from 0.5 to $2 \mathrm{hr}$. The spectra cover an approximate spectral range of $6500-9000 \AA$ at a moderate resolution (1.5 ^ FWHM) that allows the derivation of precise line-of-sight velocities. 
Table 1

Spectroscopic Objects in M87 Outer Stream Region

\begin{tabular}{|c|c|c|c|c|c|}
\hline ID & $\begin{array}{c}\text { R.A. } \\
(\mathrm{J} 2000)\end{array}$ & $\begin{array}{c}\text { Decl. } \\
(\mathrm{J} 2000)\end{array}$ & $i_{0}$ & $(g-i)_{0}$ & $\begin{array}{c}v \\
\left(\mathrm{~km} \mathrm{~s}^{-1}\right)\end{array}$ \\
\hline \multicolumn{6}{|l|}{ Confirmed } \\
\hline R8922 & 187.36271 & 12.84842 & 20.07 & 1.01 & $1460 \pm 6$ \\
\hline R10106 & 187.35946 & 12.87679 & 19.52 & 0.99 & $1500 \pm 5$ \\
\hline R10806 & 187.36816 & 12.89351 & 21.36 & 1.08 & $839 \pm 8$ \\
\hline R10814 & 187.35690 & 12.89390 & 21.38 & 0.85 & $1386 \pm 10$ \\
\hline R11987 & 187.36719 & 12.92166 & 22.24 & 0.78 & $1100 \pm 10$ \\
\hline R13629 & 187.32990 & 12.96460 & 21.65 & 0.80 & $1112 \pm 7$ \\
\hline R14045 & 187.35667 & 12.97587 & 21.72 & 0.80 & $852 \pm 8$ \\
\hline $\mathrm{R} 14100$ & 187.29791 & 12.97751 & 21.77 & 0.97 & $1154 \pm 11$ \\
\hline R15088 & 187.32974 & 13.00221 & 21.09 & 0.76 & $1395 \pm 10$ \\
\hline $\mathrm{R} 15675$ & 187.27000 & 13.01773 & 21.98 & 0.84 & $1141 \pm 18$ \\
\hline R16138 & 187.41290 & 13.02971 & 22.07 & 1.13 & $1063 \pm 12$ \\
\hline R16501 & 187.36578 & 13.03980 & 22.33 & 0.71 & $1590 \pm 12$ \\
\hline R16571 & 187.36916 & 13.04153 & 20.44 & 0.79 & $1577 \pm 9$ \\
\hline R17084 & 187.33063 & 13.05539 & 21.18 & 0.83 & $1490 \pm 8$ \\
\hline R17186 & 187.23102 & 13.05824 & 20.41 & 0.84 & $1291 \pm 6$ \\
\hline R17522 & 187.34823 & 13.06668 & 20.89 & 0.78 & $1467 \pm 5$ \\
\hline R18039 & 187.34714 & 13.08054 & 22.08 & 0.76 & $673 \pm 9$ \\
\hline R18045 & 187.31874 & 13.08087 & 21.47 & 0.81 & $1462 \pm 11$ \\
\hline R19909 & 187.33585 & 13.12520 & 21.79 & 0.83 & $1437 \pm 12$ \\
\hline $\mathrm{R} 21956^{\mathrm{a}}$ & 187.23676 & 13.16944 & 19.90 & 0.87 & $1876 \pm 7$ \\
\hline \multicolumn{6}{|l|}{ Marginal } \\
\hline $\mathrm{R} 12580$ & 187.39820 & 12.93529 & 22.01 & 0.74 & $1085 \pm 16$ \\
\hline R13657 & 187.39522 & 12.96553 & 22.14 & 0.78 & $1222 \pm 18$ \\
\hline $\mathrm{R} 15867$ & 187.41155 & 13.02209 & 22.31 & 0.75 & $1244 \pm 14$ \\
\hline R16402 & 187.32270 & 13.03704 & 22.45 & 1.21 & $898 \pm 17$ \\
\hline \multicolumn{6}{|l|}{ Stars ${ }^{\mathrm{b}}$} \\
\hline R7403 & 187.37441 & 12.81474 & 17.55 & 0.59 & $-3 \pm 48$ \\
\hline R7754 & 187.37956 & 12.82188 & 19.87 & 1.91 & $14 \pm 74$ \\
\hline R8193 & 187.38146 & 12.83134 & 19.29 & 0.69 & $-148 \pm 21$ \\
\hline R8376 & 187.37963 & 12.83570 & 20.52 & 0.98 & $-134 \pm 23$ \\
\hline R8798 & 187.39239 & 12.84514 & 18.86 & 0.72 & $65 \pm 21$ \\
\hline R9363 & 187.34117 & 12.85885 & 19.34 & 0.88 & $40 \pm 7$ \\
\hline R10146 & 187.34439 & 12.87770 & 20.45 & 0.65 & $58 \pm 20$ \\
\hline R10837 & 187.36675 & 12.89449 & 17.67 & 2.65 & $42 \pm 14$ \\
\hline R11062 & 187.40232 & 12.89997 & 15.38 & 1.74 & $-24 \pm 5$ \\
\hline R11805 & 187.39709 & 12.91762 & 22.47 & 1.33 & $189 \pm 20$ \\
\hline R11872 & 187.36193 & 12.91931 & 19.96 & 1.11 & $45 \pm 15$ \\
\hline $\mathrm{R} 12125$ & 187.40527 & 12.92458 & 20.42 & 0.74 & $145 \pm 6$ \\
\hline $\mathrm{R} 12313$ & 187.29011 & 12.92963 & 15.75 & 0.49 & $4 \pm 20$ \\
\hline R12657 & 187.34624 & 12.93754 & 17.48 & 1.09 & $10 \pm 5$ \\
\hline R12691 & 187.40031 & 12.93805 & 16.32 & 2.03 & $-20 \pm 5$ \\
\hline R12891 & 187.34345 & 12.94330 & 20.44 & 0.84 & $177 \pm 51$ \\
\hline R13164 & 187.30174 & 12.95084 & 20.11 & 0.69 & $-41 \pm 25$ \\
\hline R13188 & 187.23932 & 12.95184 & 17.91 & 1.33 & $35 \pm 17$ \\
\hline R13506 & 187.46370 & 12.95930 & 15.37 & 0.35 & $-6 \pm 8$ \\
\hline R13992 & 187.33976 & 12.97469 & 19.83 & 1.80 & $4 \pm 36$ \\
\hline R14054 & 187.45815 & 12.97566 & 19.07 & 0.74 & $98 \pm 5$ \\
\hline R14166 & 187.33685 & 12.97877 & 20.88 & 1.03 & $198 \pm 7$ \\
\hline $\mathrm{R} 14317$ & 187.34125 & 12.98226 & 15.49 & 0.92 & $30 \pm 5$ \\
\hline R14391 & 187.35850 & 12.98435 & 19.70 & 0.69 & $-87 \pm 6$ \\
\hline R14427 & 187.44737 & 12.98488 & 20.96 & 1.03 & $213 \pm 14$ \\
\hline $\mathrm{R} 14800$ & 187.26911 & 12.99484 & 15.70 & 1.67 & $0 \pm 18$ \\
\hline R15119 & 187.40424 & 13.00259 & 17.83 & 2.02 & $9 \pm 5$ \\
\hline R15179 & 187.44657 & 13.00379 & 17.83 & 0.87 & $70 \pm 5$ \\
\hline R15494 & 187.45031 & 13.01196 & 19.09 & 0.82 & $-3 \pm 5$ \\
\hline R15721 & 187.30143 & 13.01873 & 16.86 & 0.59 & $58 \pm 18$ \\
\hline R15838 & 187.42486 & 13.02142 & 21.09 & 0.72 & $-31 \pm 10$ \\
\hline R15983 & 187.36178 & 13.02593 & 21.37 & 1.89 & $55 \pm 32$ \\
\hline R16488 & 187.39562 & 13.03932 & 19.52 & 1.12 & $108 \pm 5$ \\
\hline $\mathrm{R} 17262$ & 187.41103 & 13.05953 & 22.24 & 0.64 & $110 \pm 24$ \\
\hline R17841 & 187.35704 & 13.07556 & 22.00 & 0.72 & $185 \pm 12$ \\
\hline R18139 & 187.27810 & 13.08366 & 22.26 & 1.27 & $-84 \pm 26$ \\
\hline $\mathrm{R} 18154$ & 187.34945 & 13.08374 & 18.48 & 1.54 & $-57 \pm 5$ \\
\hline
\end{tabular}

Table 1

(Continued)

\begin{tabular}{lccccc}
\hline \hline ID & $\begin{array}{c}\text { R.A. } \\
(J 2000)\end{array}$ & $\begin{array}{c}\text { Decl. } \\
(\text { J2000 })\end{array}$ & $i_{0}$ & $(g-i)_{0}$ & $\begin{array}{c}v \\
\left(\mathrm{~km} \mathrm{~s}^{-1}\right)\end{array}$ \\
\hline R18730 & 187.27131 & 13.09786 & 21.26 & 0.80 & $-214 \pm 15$ \\
R19052 & 187.27947 & 13.10543 & 15.40 & 0.97 & $-35 \pm 15$ \\
R19452 & 187.37246 & 13.11475 & 16.77 & 2.38 & $-1 \pm 5$ \\
R20070 & 187.26784 & 13.12938 & 17.82 & 0.67 & $127 \pm 14$ \\
R20445 & 187.30037 & 13.13758 & 20.15 & 0.76 & $103 \pm 6$ \\
R20496 & 187.38952 & 13.13803 & 21.20 & 0.78 & $32 \pm 16$ \\
R20765 & 187.24172 & 13.14418 & 20.54 & 0.72 & $222 \pm 18$ \\
R21009 & 187.23749 & 13.14917 & 15.18 & 1.85 & $5 \pm 17$ \\
R21082 & 187.29307 & 13.15058 & 17.69 & 1.30 & $-6 \pm 8$ \\
R22195 & 187.33737 & 13.17345 & 20.94 & 0.79 & $73 \pm 9$ \\
R22421 & 187.33604 & 13.17731 & 21.49 & 0.57 & $143 \pm 16$ \\
R22843 & 187.32868 & 13.18426 & 20.83 & 0.71 & $22 \pm 13$ \\
Galaxies & & & & & \\
R12858 & 187.23866 & 12.94289 & 22.24 & 0.71 & $\ldots$ \\
R13471 & 187.25251 & 12.95961 & 21.89 & 1.05 & $\ldots$ \\
R14229 & 187.22466 & 12.98077 & 22.03 & 0.94 & $\ldots$ \\
R15594 & 187.25608 & 13.01557 & 22.41 & 0.98 & $\ldots$ \\
R15685 & 187.23852 & 13.01819 & 22.40 & 1.37 & $\ldots$ \\
R17134 & 187.23876 & 13.05692 & 22.59 & 0.77 & $\ldots$ \\
R21550 & 187.21381 & 13.15999 & 22.97 & 0.69 & $\ldots$ \\
\hline & & & & & $\ldots$ \\
\hline
\end{tabular}

Notes. Photometry is from Subaru-Suprime/Cam imaging using four-pixel aperture magnitudes, bootstrapped to the Sloan Digital Sky Survey and then corrected for Galactic extinction following Peek \& Graves (2010). The uncertainties in the stellar velocities are indicative only and not as carefully characterized as for the GCs. The most likely objects belonging to the cold GC stream are R16501, R16571, R17084, R17522, and R18045.

a Probably bound to NGC 4461.

b A simple velocity boundary of $250 \mathrm{~km} \mathrm{~s}^{-1}$ has been used to separate stars and GCs, but some of the higher-velocity stars might in principle be low-velocity GCs.

(This table is also available in a machine-readable form in the online journal.)

The data were reduced in a standard manner, including bias subtraction, flat fielding, wavelength calibration, and sky subtraction, followed by optimal extraction. Heliocentric lineof-sight velocities were derived through cross-correlation with a range of stellar templates, using only the region around the Calcium II triplet (CaT; $\mathrm{H} \alpha$, if available, was used for confirmation of borderline cases). Some background galaxies were identified via examination of the extracted spectra and excluded from further analysis.

The velocity uncertainties were estimated by combining in quadrature the statistical uncertainties from the cross-correlation with the systematic uncertainties from using different stellar templates. The uncertainties for the confirmed GCs ranged from 5 to $18 \mathrm{~km} \mathrm{~s}^{-1}$, with their reliability supported by repeat observations of four GCs and five stars. The characteristics of the spectroscopically confirmed GCs (20 of them), stars, and galaxies in the stream region are provided in Table 1. We also targeted five PN candidates from Feldmeier et al. (2003) but found no $\mathrm{H} \alpha$ emission in their spectra (objects 7-14, 7-18, 7-51, 7-58, 7-65).

Our new spectroscopic data thus provides a total of 468 velocities of M87 GCs (we exclude one large-radius object probably associated with the Virgo galaxy NGC 4461, but retain another that may be bound to the small elliptical NGC 4478). Although a great deal of additional spectroscopic data of M87 GCs are available in the literature, we do not use most of the previous data, since the typically large measurement errors impede the study of cold substructures. Instead, we incorporate only 20 high-resolution measurements from the literature (with 
median uncertainties of $4 \mathrm{~km} \mathrm{~s}^{-1}$; Haşegan et al. 2005; Haşegan 2007; Evstigneeva et al. 2007). Note that our treatment of previous data differs slightly from the convention of $\mathrm{S}+11$, who incorporated all of the data published since 2003.

The combined literature and new data provide a total sample of 488 GC line-of-sight velocities around M87, extending from its central regions out to $200 \mathrm{kpc}$ in its halo, where the median velocity uncertainties are $14 \mathrm{~km} \mathrm{~s}^{-1}$. This contrasts with most previous work on GC kinematics beyond the Local Group, with typical velocity errors of $\sim 30-100 \mathrm{~km} \mathrm{~s}^{-1}$. Our GC sample also has an extremely low rate of contamination from foreground stars and background galaxies. ${ }^{7}$ This is the largest GC data set of this quality in any galaxy, providing an unprecedented opportunity for exploring phase space for formational relics.

We present a basic overview of the GC observations in Figures 1(a) and (b), with a plot of positions in real space along with a phase-space plot of velocity versus galactocentric radius. It is important to note that most of the spatial substructure apparent in the GC data is just a product of inhomogeneous spectroscopic sampling, e.g., the "gap" at a radius of $\sim 7 \mathrm{arcmin}$, and elongated extensions to the far east and northwest. Our analyses will be relatively insensitive to such inhomogeneities since we will focus on the distribution of velocities as a function of galactocentric radius. Furthermore, the sampling effects will be built in to our statistical tests for substructure.

Most of the GCs show a broad spread of velocities reflecting a dynamically relaxed system, but there are two sharper features that stand out in phase space. The first is a small elongated "stream" of GCs at $150 \mathrm{kpc}$, associated with the known stellar filament, the second is a large chevron-shaped overdensity between 50 and $100 \mathrm{kpc}$, which we denote as the "shell." It is an enormous structure spread out over $\sim 10^{4} \mathrm{kpc}^{2}$ on the sky, and is not identifiable with any previously known photometric feature (Figure 1(a)). The statistical significance of these two substructures will be demonstrated in Sections 3 and 4, where we also find independent support from their distinct GC metallicity distributions.

Both features are most naturally explained as the shredded remnants of infalling galaxies, which we will investigate in Section 5 using numerical simulations. To orient the discussion of the rest of the paper, we present illustrative results from these simulations in Figure 2, which broadly reproduce some of the general features of the data.

\section{INNER SHELL ANALYSIS}

Here we characterize the properties of the large shell identified in Figure 1(b) and attempt to establish its statistical significance. We exclude the outer stream data set from these analyses, thereby focusing on the main part of the data set which has relatively unbiased spatial sampling.

In Section 3.1, we compare the use of different conventions for radial distance. We carry out a group-finding analysis and quantify the entropy in the phase-space data in Sections 3.2 and 3.3 , and perform maximum-likelihood fitting of simple shell models in Section 3.4. In Section 3.5 we analyze the shell GC

\footnotetext{
7 Our sample of "GCs" includes some mysterious objects termed ultracompact dwarfs, which have sizes of $\sim 10 \mathrm{pc}$ or more, in contrast to classical GCs with sizes of a few pc. Whether or not there are two or more classes of object, or a continuum of GCs with different properties, is a major scientific question that we cannot resolve here (see Brodie et al. 2011). Therefore, although we know for a small subset of our spectroscopic sample that the sizes are unusually large, we retain these in any case as tracer particles in the halo (excluding only S923 which has a peculiar morphology).
}

color distributions, and in Section 3.6 consider the implications for the shell progenitor.

\subsection{Radius Convention}

One of the greatest challenges in detecting and analyzing substructures in extragalactic systems is decoding the multi-dimensional information contained in observations. The dynamical definition of a substructure is that it occupies a distinct region in some phase space of physical parameters that are linked to orbital trajectories. This could be a space defined by the integrals of motion, such as the energy and angular momentum in a spherical gravitational potential, or it could be the six-dimensional observational phase space of position and velocity.

To pick out a substructure from a background of unrelated structures, it helps to link the observations to orbital solutions. This can be tricky enough with six-dimensional data as available for some objects within the Milky Way, but in distant galaxies, only three dimensions are normally observable (two of position and one of velocity). This "projection" of phase space typically smears out the orbital tracks and can make substructure inferences degenerate.

If one already knew a priori the orbital characteristics of a substructure, one could apply an optimal coordinate transformation to the observations that would maximize the contrast in a "reduced" phase-space plot. One of the most basic reductions to try is converting the two spatial positions to a galactocentric radius, since in the limit of a spherical potential and either a radial or a circular orbit, the particles will outline a characteristic chevron-shaped track in the phase space of radius and line-of-sight-velocity (cf. Figure 1 of Rix et al. 1997).

Real galaxies are not perfectly spherical, and in dynamical modeling there is a standard modification to the above transformation that corrects for flattening to first order. This is to use an ellipse-based circular-equivalent or "intermediate-axis" radius. If $x$ and $y$ are projected coordinates along a galaxy's major and minor axes, respectively, then the simple projected radius is

$$
R_{\mathrm{p}}=\sqrt{x^{2}+y^{2}} .
$$

The equivalent radius, given an axis ratio $q$, is

$$
R_{m}=\sqrt{q x^{2}+\frac{y^{2}}{q}} .
$$

This convention allows one to conserve the area (and approximately the mass) enclosed within an elliptical isophote when transforming to a circularized one-dimensional model. It is also equivalent to recovering a constant radius $r$ in three dimensions for an inclined thin disk.

The next question is what value for $q$ to use, as both the flattening of the observable objects and of the unobserved gravitational potential could be important. Unfortunately, in the outskirts of M87, neither of the flattenings is well constrained (i.e., for the GC system or for the potential which is dominated by dark matter). It was found in $\mathrm{S}+11$ (Figure 7) that the GC system has a typical $q \sim 0.75$ at $R_{m} \sim 2^{\prime}-10^{\prime}(\sim 5-50 \mathrm{kpc})$, while possibly decreasing with radius. At larger radii, the measurement is more difficult, but we have used a maximumlikelihood technique to estimate a possible range of $q \sim$ $0.55-0.75$, at $R_{m} \sim 15^{\prime}-20^{\prime}(\sim 70-100 \mathrm{kpc})$.

Our constraints on $q\left(R_{m}\right)$ for the GCs are consistent with the profile for the stars derived by Kormendy et al. (2009), where $q$ 

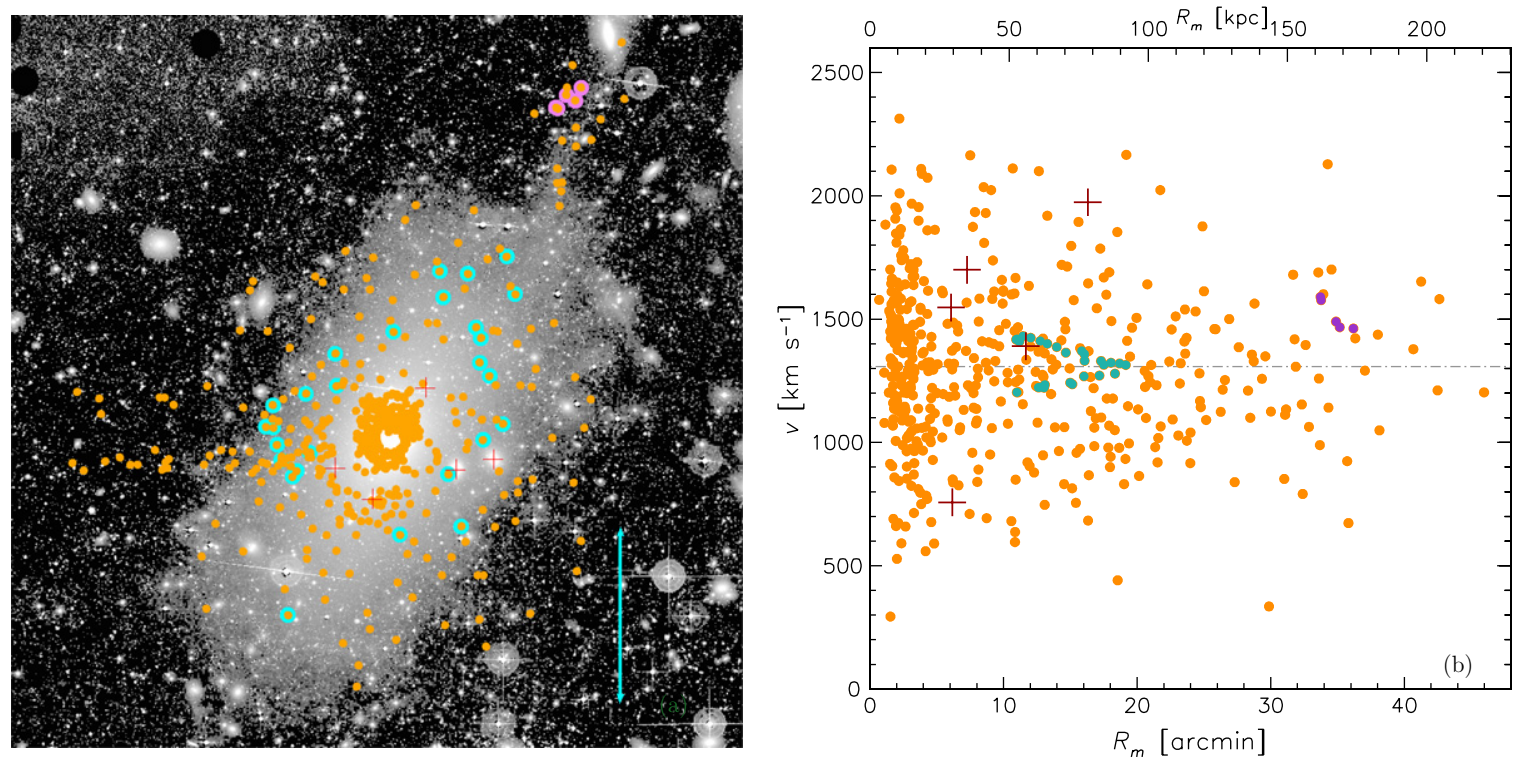

Figure 1. Overview of spectroscopic GC observations around M87. Orange circles show confirmed GCs, red crosses show five centrally located low-luminosity elliptical galaxies, and candidate substructure members are highlighted with blue and purple. These are an inner shell (at radii of $\sim 10-20$ arcmin) and an outer stream (at $\sim 35$ arcmin), where the most likely members are identified by maximum-likelihood fitting to simple models (see Section 3.4). (a) Data locations in positional space, overplotted on a deep optical image (down to a $V$-band surface brightness of $\mu_{V} \sim 28.5 \mathrm{mag} \mathrm{arcsec}-2$; Janowiecki et al. 2010), with a $100 \mathrm{kpc}$ (21 arcmin) scale illustrated by a bar with arrows. (b) The phase space of line-of-sight velocity vs. galactocentric radius, with the dot-dashed horizontal line representing the systemic velocity of M87 $\left(1307 \mathrm{~km} \mathrm{~s}^{-1}\right)$. The radius plotted is the intermediate-axis radius (see Section 3.1). The velocity uncertainties are not shown, but are generally smaller than the point sizes in the plot. The satellite galaxy velocities are from Hypercat (Paturel et al. 2003), where we note that the value for NGC 4486A was recently dramatically revised by Prugniel et al. (2011).

(A color version of this figure is available in the online journal.)
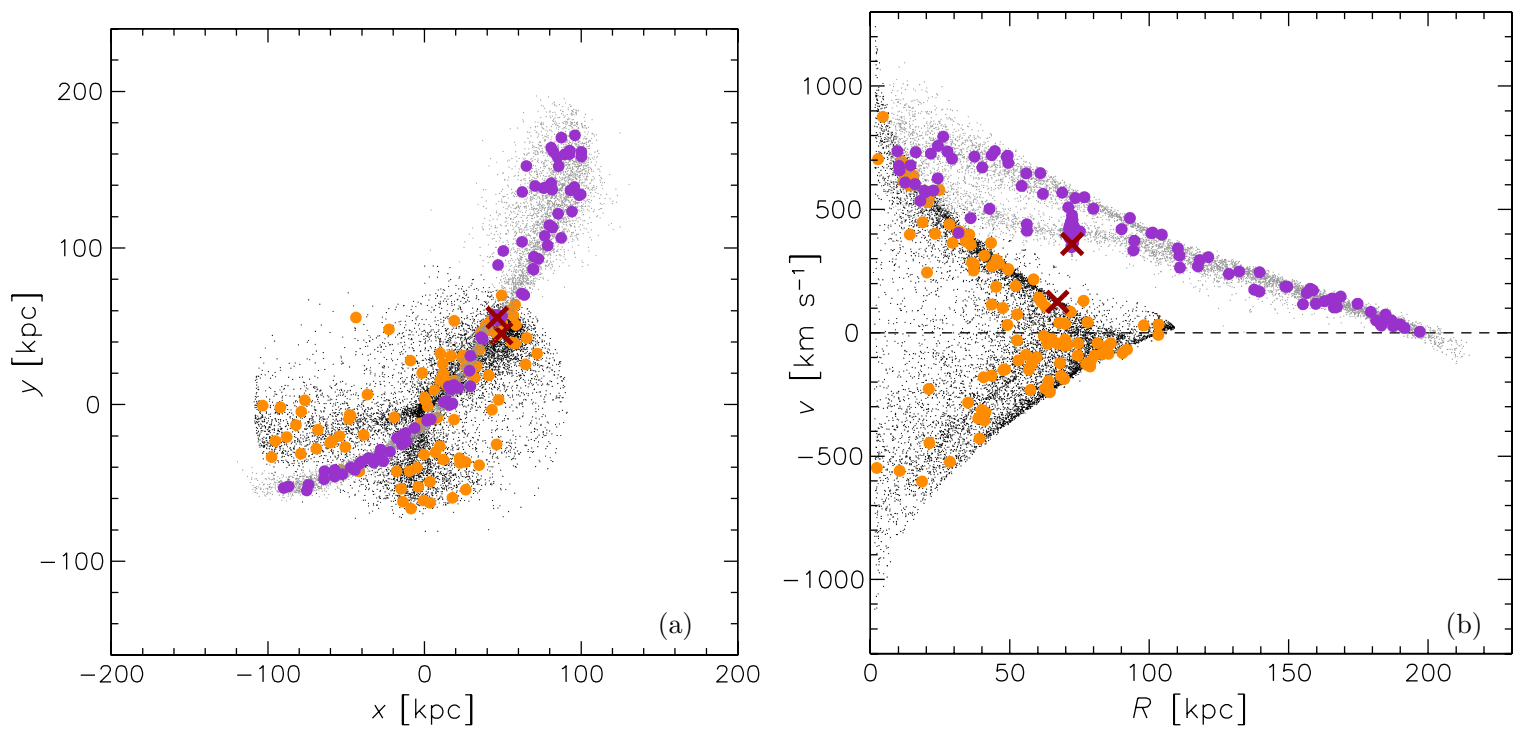

Figure 2. Simulations of galaxies falling into an idealized central cluster potential, as in Figure 1, both positional space (a) and phase space (b) are shown. In each simulation, subsamples of 100 and $10^{4}$ particles are plotted as large and small dots, respectively. The red $\times$ symbols show the locations of the progenitor nuclei. Two independent simulations are superimposed, representing initial apocentric distances of 90 and $200 \mathrm{kpc}$ (using small black and large orange dots, and small gray and large purple dots, respectively). These two cases are intended to be qualitative analogs to the M87 shell and stream (Figure 1), with the $0.2 \%$ particle subsamples as "GCs" (with small measurement errors added). The snapshots shown correspond to $3.5 \mathrm{Gyr}(\sim 10-20$ dynamical times) after the initial apocenters. In the first case, there are sharp features in phase space even when the tidal debris is well mixed in positional space (which happens more rapidly for a smaller initial apocenter). See Section 5 for further details.

(A color version of this figure is available in the online journal.)

declines from $\sim 0.9$ at $R_{m} \sim 1^{\prime}$ to $\sim 0.55$ outside $R_{m} \sim 10^{\prime}$. We therefore adopt these stellar results for our default model for the GCs, for the sake of having smooth, plausible profiles of $q$ and position angle with radius. ${ }^{8}$

8 A more recent study of M87 with deeper surface photometry found that the stellar-light flattening becomes even stronger at large radii, reaching $q \sim 0.4$ at $R_{m} \sim 20^{\prime}$ (Janowiecki et al. 2010). This is clearly inconsistent with the flattening of the GC system, and it would be inappropriate to use this profile for our analyses. Both the Kormendy and the Janowiecki profiles agree on a position angle of $\sim 150^{\circ}$ at large radii, while our GC analysis is more suggestive of $\sim 105^{\circ}-135^{\circ}$. We do not ascribe much credence to this inconsistency, given the difficulties with background contamination and spatial incompleteness at large radii, and given the much better consistency between stars and GCs at small radii. 

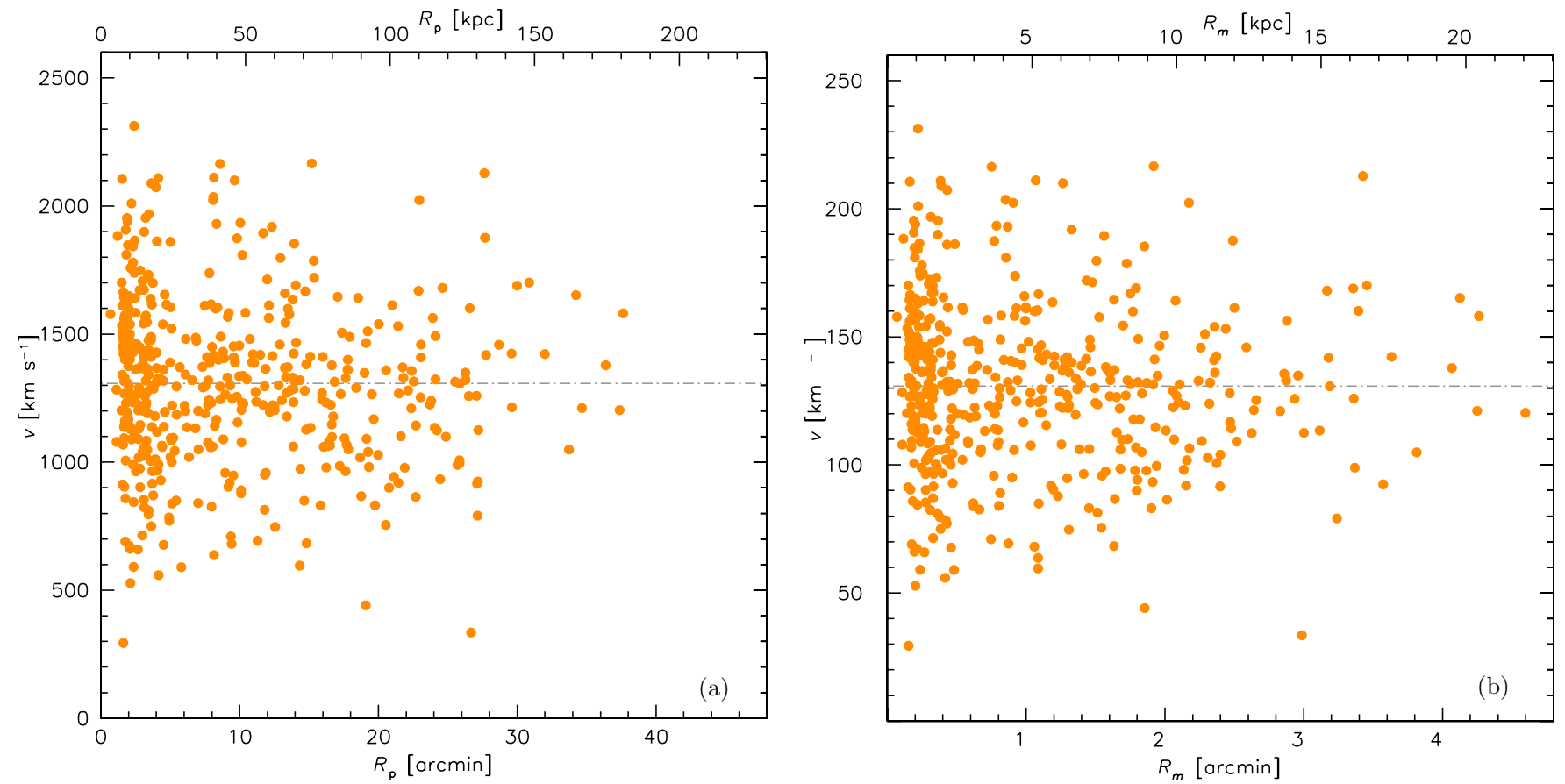

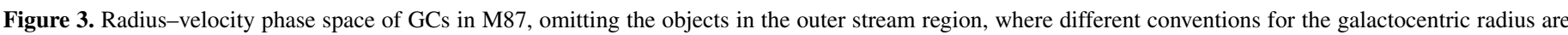

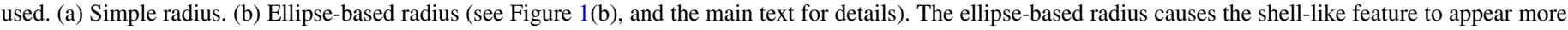
distinct.

(A color version of this figure is available in the online journal.)

Figure 3 then compares radius-velocity phase-space plots for the M87 GCs, using both the simple and the elliptical radius. The cold shell at $\sim 50 \mathrm{kpc}$ appears considerably sharper when using the elliptical radius, and we suspect that it may be easier to find substructures in other data sets if the $R_{m}$ convention is used.

We will adopt this $R_{m}$ transformation as our default for the analyses used in the rest of this paper, while issuing some caveats. One is that it is not entirely clear from a theoretical standpoint why the transformation should work: an accretion event generally defines its own orbital plane that will not necessarily align with the apparent shape of the rest of the galaxy. It could be that we are seeing an effect related to the disrupted accretor being highly spread out in the host potential.

The other concern from an observational standpoint is that the $q\left(R_{m}\right)$ profile for the GCs is not well determined, and fairly small changes in the radius transformation (of $\sim 1^{\prime}$ ) would noticeably affect the inferred morphology of the substructure, causing it to lose some of its apparent sharpness in phase space. Such "blurring" would be expected if there is a genuine cold feature, and if one deviates from an optimal coordinate transformation as discussed above (we have verified this effect using mock data simulations that we will discuss later). However, we consider it more difficult to artificially sharpen phase-space features through a transformation error.

In summary, the choice of the radius in the phase-space diagram is a source of systematic uncertainty that is difficult to quantify. Where relevant in our analyses, we will also consider the possibility that the substructure is more amorphous than in our default model (e.g., as in Figure 3(a)).

\subsection{Group Finding}

To ascertain the veracity of the shell, rather than rely only on the notoriously misled human eye which is good at creating patterns out of random noise, we examine several different types of models and statistical tests. This is an aspect that has been underdeveloped in previous work on GC kinematics around early-type galaxies.

Our first step is to use a group-finding algorithm, based on a standard friends-of-friends approach (e.g., Perrett et al. 2003; Rudick et al. 2009). We define a distance between objects of

$$
\Delta s=\overline{\left(\frac{\Delta \log _{10} R_{m}}{w_{R}}\right)^{2}+\left(\frac{\Delta v}{w_{v}}\right)^{2}},
$$

where $w_{R}$ and $w_{v}$ are radius and velocity scale factors that act inversely as weights. Note that our adopted two-dimensional phase-space metric differs from the three-dimensional $(x, y, v)$ metric commonly used, which is suited for very localized substructures that have had little mixing, but we find that it does not work well for structures like shells and long streams that have elongated "diagonal" morphologies in phase space. Using the three-dimensional metric may have caused large-scale substructures to be missed in the past.

A group is defined as a set of objects separated by less than a linking length $\Lambda$ in phase space, which is in turn defined relative to the mean interparticle spacing $\langle\Delta s\rangle$ by a dimensionless parameter $\lambda$. We then have the criterion

$$
|\Delta s|<\Lambda=\lambda\langle\Delta s\rangle .
$$

The next step is to choose the free parameters $\left(w_{R}, w_{v}, \lambda\right)$ in the algorithm, where the objective is to maximize the sensitivity to real features in the data while minimizing the incidence of false groups and linkages. As a starting point, we construct mock data sets based on simulations of an accretion event (which we will discuss in detail later). To represent the shell, we select 47 particles, a number that is chosen to mimic the real data set, in the sense that the simulation has 27 particles in the same 


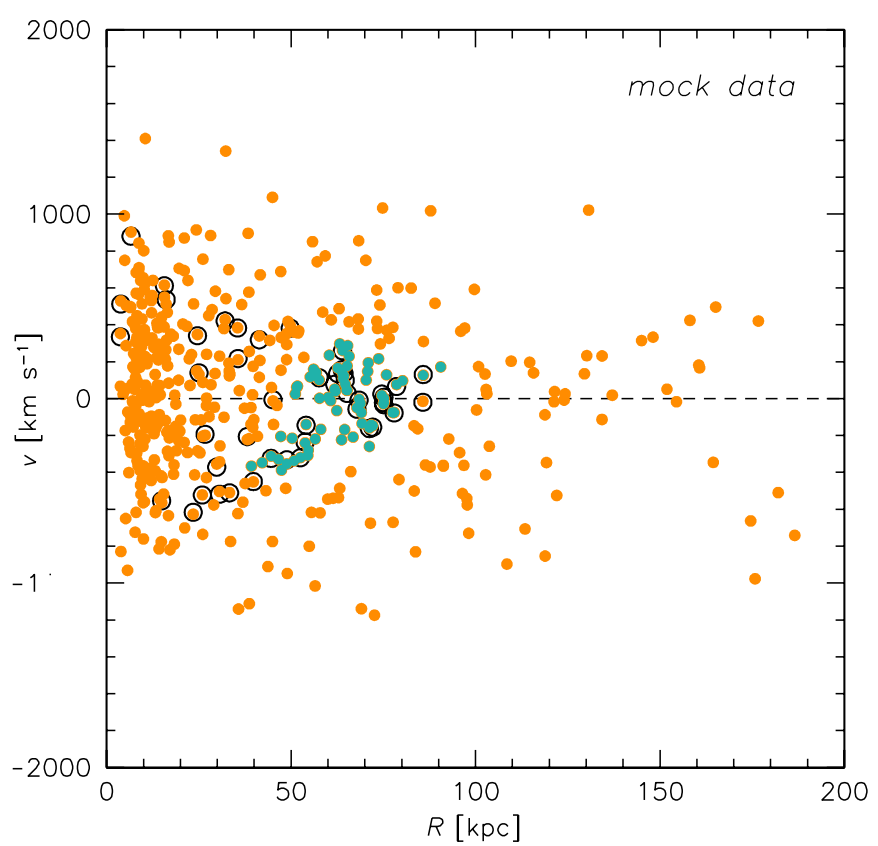

Figure 4. Phase-space group finding in a mock data set constructed from a simulated accretion event (see Figure 2(b)) superimposed on a randomized population of GCs. The genuine shell objects are shown as black circles, with velocities that differ slightly from the mock data set because of simulated observational errors. The general morphology of the substructure is recovered by the group-finding algorithm (blue dots). Compare application to the real data in Figure 1(b).

(A color version of this figure is available in the online journal.)

radial region where we find a shell in the real data (which has an estimated 27 GCs as we will see later).

We then fill out the rest of the mock data set by adding 422 GCs drawn from a Gaussian velocity distribution with $\sigma=450 \mathrm{~km} \mathrm{~s}^{-1}$, and with positions corresponding to those in the real data set, but randomized by $20 \%$ in order to reduce any lingering spatial substructures. Throughout this paper, we will construct similar, smooth "background" GC populations, which would be unrealistic at some level if the halo were rich in substructures of various masses and stages of dissolution. In the future, one could consider constructing background distributions based on a range of theoretical models for halo accretion histories, but for the purposes of this paper, we focus primarily on the null hypothesis of a halo with no substructure. A caveat to then keep in mind is that the large "shell" which we recover could in principle consist of two or more distinct substructures that overlap in the observable phase space and conspire to masquerade as a single structure.

Note that the value of $\sigma=450 \mathrm{~km} \mathrm{~s}^{-1}$ is larger than the $\sim 300 \mathrm{~km} \mathrm{~s}^{-1}$ of the real data, which is done to be consistent with the simulations' circular velocity and larger shell velocity width. We will later rescale the resulting group-finding parameters to suit the real data. The exact velocities of the mock data set are also convolved with small errors corresponding to the observational uncertainties in the real data.

We find optimum parameters for the simulated data set of $w_{R}=0.035 \mathrm{dex}, w_{v}=50 \mathrm{~km} \mathrm{~s}^{-1}$, and $\lambda=0.07$, with acceptable variations around these values at the $\sim 20 \%$ level. The group-finding results are shown in Figure 4: a group of 70 objects is identified, which reproduces the broad morphology of the shell's limb (cf. Figure 2(b)) and recovers many of the genuine shell members along with many false associations. This exercise provides some confidence in the simple group-finding approach for recovering cold phase-space substructures that comprise only $\sim 10 \%$ of a sample. However, the individual objects assigned to the substructure can include a high degree of contamination. This fact, combined with the potential sensitivity of the group-finding parameters to the details of the data sampling and of the substructure properties, motivates exploring alternative algorithms (which we do below) as well as the future development of more efficient phase-space group finders.

Now after rescaling the foregoing simulation-based parameters, we adopt the following for use with the real data: $w_{R}=0.035 \mathrm{dex}, w_{v}=30 \mathrm{~km} \mathrm{~s}^{-1}$, and $\lambda=0.07$. The groupfinding algorithm then recovers a shell-like substructure with 68 members, around half of which may be false associations, which we illustrate with Figure 5(a). Given the uncertainty in the radius convention discussed in Section 3.1, we also carry out the same procedure while using the simple radius $R_{\mathrm{p}}$. We find in this case a group with 80 members demonstrating that our detection of a large phase-space substructure in M87 is not strongly sensitive to the radius convention.

To further evaluate the statistical significance of this shell, we carry out Monte Carlo simulations of underlying smooth data sets. We generate 100 mock GC data sets, using in each case the same radial locations as in the real data, but substituting a random velocity drawn from a Gaussian distribution with $\sigma=324 \mathrm{~km} \mathrm{~s}^{-1}$ (which is the dispersion found in the real data).

We then run the group-finding algorithm with the same parameters as for the real data. The median largest group size found (outside the crowded central regions where the groupfinding parameters are not optimized) has 30 members, and the largest group of all has 61 members. Examples of these groups are shown in panels (b) and (c) of Figure 5, alongside the real data in panel (a). We thus exclude finding a 68-member group by chance at the $99 \%$ level. This test is somewhat conservative in using the radial locations of the real GCs in the mock data sets, which may already have some clumpiness.

If we were to include the data from the outer stream region, a stream-like phase-space feature would indeed be picked up by the group-finding algorithm, but so would be six other small "substructures" that (based on the Monte Carlo simulations above) are probably not real. The outer stream is analyzed separately in a later section, using a slightly different algorithm because of the much smaller number counts and the importance of two-dimensional spatial clumping.

\subsection{Entropy}

The next test uses a very general entropy metric $S$ that measures the clumpiness of data in a set of partitions:

$$
S=-\sum_{i} N_{i} \ln N_{i}
$$

where $N_{i}$ is the number of objects in each partition (Helmi et al. 1999). We consider the 4-20 arcmin region of interest and adopt a gridded partition of (radius by velocity) with size ( $2 \operatorname{arcmin} \times 80 \mathrm{~km} \mathrm{~s}^{-1}$ ). We measure $S$ and compare it to 1000 mock data realizations using Gaussian velocity distributions. We find that this region is significantly substructured at the $1 \sigma$ level. This is a conservative test because again we have used radii from the real data, and because the rectangular partitioning is not optimized to pick up extended diagonal features as the data appear to show. 


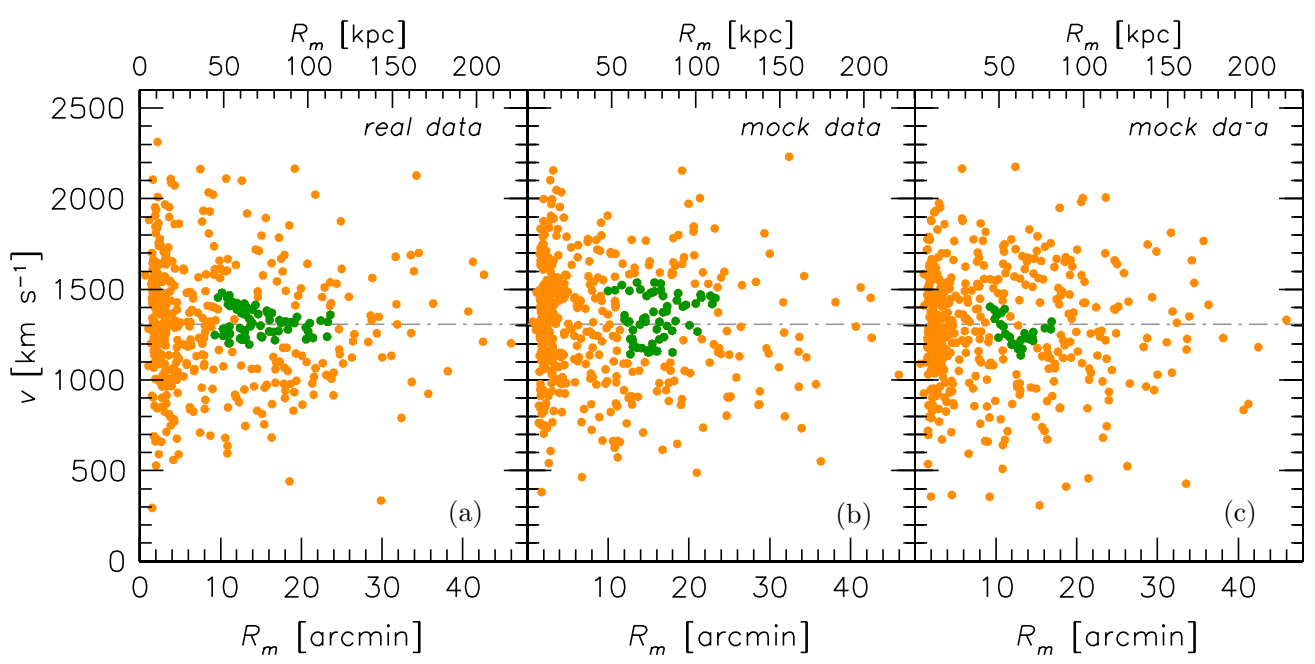

Figure 5. Group finding of GCs in phase space. Orange dots show the parent GC population, while green dots show the largest identified groups in each data set. (a) The real M87 data (where the outer stream region is again omitted). (b) The mock data set generated from an unstructured model, with the largest "group" marked. (c) A typical mock data set. The false group detections typically have many fewer objects than in the M87 data, and lack the clear, fairly symmetric shell-like morphology. (A color version of this figure is available in the online journal.)

\subsection{Maximum Likelihood Modeling}

We next carry out a set of parametric model fits to the data in the shell region, which both allows us to evaluate the significance of the substructure and to quantify its properties. We will in general focus on the $R_{m}=10.5-20$ arcmin $(50-95 \mathrm{kpc}$ ) region where the shell is most apparent by eye.

The basic idea is that given a model for the line-of-sight velocity distribution (LOSVD) $d L / d v$ at a given measurement location $R_{i}$, we quantify the likelihood $\mathcal{L}$ of a velocity measurement $v_{i} \pm \Delta v_{i}$ :

$$
\mathcal{L}\left(v_{i}, R_{i}\right)=\int_{-\infty}^{\infty} \frac{d L}{d v}\left(v, R_{i}\right) e^{-\frac{\left(v-v_{i}\right)^{2}}{2\left(\Delta v_{i}\right)^{2}}} d v
$$

(Romanowsky \& Kochanek 2001). The likelihood can be considered as equivalent to a $\chi^{2}$ statistic after the operation $-2 \ln \mathcal{L}$. For a Gaussian model $d L / d v$ with an intrinsic dispersion $\sigma$ centered on a systemic velocity $v_{\text {sys }}$, the maximum-likelihood model fitting involves minimizing the simple function:

$$
-2 \ln \mathcal{L}=\frac{\left(v_{i}-v_{\text {sys }}\right)^{2}}{\sigma^{2}+\left(\Delta v_{i}\right)^{2}}+\ln \left[\sigma^{2}+\left(\Delta v_{i}\right)^{2}\right]
$$

(Nolthenius \& Ford 1986; Bergond et al. 2006).

Before constructing detailed models of the system in phase space, we consider the simplest possible models, lumping all of the shell-region velocity data together, with no spatial information. Using the likelihood formalism above, we fit a single Gaussian to these data and find a dispersion of $315 \mathrm{~km} \mathrm{~s}^{-1}$. The implied LOSVD for this model is shown along with the data in Figure 6(a).

It is apparent that a single Gaussian is not a good representation of the data, so we next try a double-Gaussian model. We find a best fit where the hot component has a dispersion of $361 \pm 34 \mathrm{~km} \mathrm{~s}^{-1}$, and the cold component comprises a fraction $f_{\mathrm{s}}=0.25 \pm 0.12$ of the GCs, with a dispersion of $86 \pm 34 \mathrm{~km} \mathrm{~s}^{-1}$ (where Monte Carlo simulated data sets were used to estimate these uncertainties and to correct for slight fitting biases). We show a mock data set of this solution in Figure 6(a) to illustrate the level at which noise can influence the LOSVD.
By eye, the second Gaussian appears to be required for adequately fitting the data, and to quantify this statement we carry out a Monte Carlo analysis using a series of singleGaussian mock data sets. We find that $f_{\mathrm{s}}$ values as high as 0.24 are fitted by chance in only 15 out of 1000 simulations, while most of these false second components are hot, with only 1 out of 1000 having a dispersion as low as $\sim 80 \mathrm{~km} \mathrm{~s}^{-1}$. We conclude that there is definitely a secondary cold component, with a dispersion of $120 \mathrm{~km} \mathrm{~s}^{-1}$ at the most, comprising $13 \%-37 \%$ of the GC data. It is also important to note that this conclusion does not depend on the detailed morphology of the substructure and is insensitive to the adopted radius convention (Section 3.1), since we have not used radius information except to define the overall radial bin, and the results are also very similar if we define this bin using the circular radius $R_{\mathrm{p}}$.

Next, given the radial dependence of the shell that is visible by eye in Figure 3(b), we examine the LOSVD in two radial bins: one at the "mouth" of the substructure and one at the "apex" (Figures 6(a) and (b)). We again fit double-Gaussian models, allowing for a split around $v_{\text {sys }}$ for the cold component. In the first bin, we find a dispersion of $23_{-14}^{+15} \mathrm{~km} \mathrm{~s}^{-1}$ with $f_{\mathrm{s}}=0.30_{-0.12}^{+0.13}$ and a peak separation of $214 \pm 22 \mathrm{~km} \mathrm{~s}^{-1}$. In the second, we find $f_{\mathrm{s}}=0.12_{-0.07}^{+0.10}$ and a dispersion consistent with zero with a $68 \%$ upper limit of $20 \mathrm{~km} \mathrm{~s}^{-1}$.

These binned results together suggest a cold substructure with $f_{\mathrm{s}} \sim 0.2$ and a dispersion of $\sim 15 \mathrm{~km} \mathrm{~s}^{-1}$. The latter result is similar to our rms velocity measurement errors, which can be challenging to determine accurately, and consequently, a dispersion anywhere in the range $0-35 \mathrm{~km} \mathrm{~s}^{-1}$ is plausible.

We now model all of the shell-region data jointly in phase space. Each model consists of a fraction $f_{\mathrm{s}}$ of substructure, and a Gaussian component with fraction $\left(1-f_{\mathrm{s}}\right)$ and dispersion $\left(\sigma_{1}\right)$. The substructure LOSVD is parameterized in one of three ways: as a single Gaussian, a nested double Gaussian, or a step function. These models are all combined with a linear trend of LOSVD width with radius, producing a tapered Gaussian, a chevron, or a filled wedge, respectively. The characteristic width versus radius is

$$
v_{\mathrm{w}}=v_{\mathrm{sys}} \pm v^{\prime} \times\left(R-R_{\mathrm{x}}\right),
$$



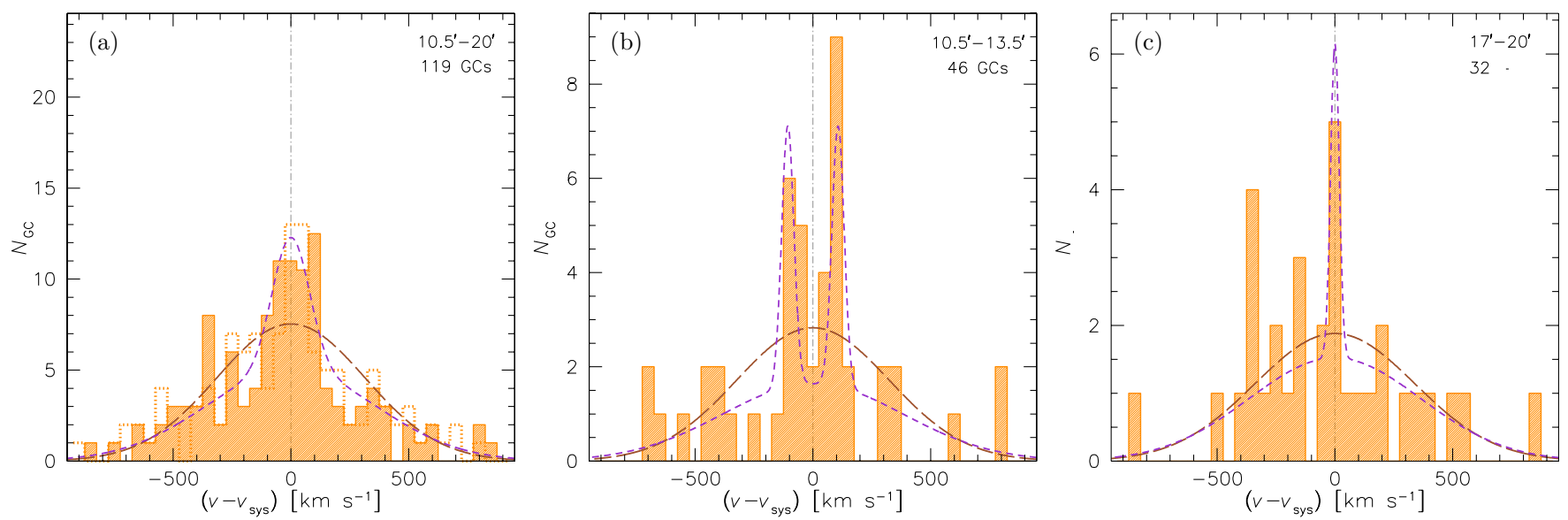

Figure 6. Line-of-sight velocity distribution of GCs in the shell region around M87 ( $R_{m}=10.5-20$ arcmin). The systemic velocity of $1307 \mathrm{~km} \mathrm{~s}^{-1}$ has been subtracted from the data. The filled histograms show the data, while the long- and short-dashed curves illustrate single- and double-Gaussian model fits, respectively (with additional smoothing to account for measurement errors). The panels show different radial regions. (a) Entire region. A mock data set is also shown as a dotted histogram. (b) and (c) Finer radial bins as labeled. The shell-like substructure is clearly visible as an extra, low-dispersion component in the overall velocity distribution. At smaller radii, it appears as a symmetric double peak, and at larger radii as a narrow single peak.

(A color version of this figure is available in the online journal.)

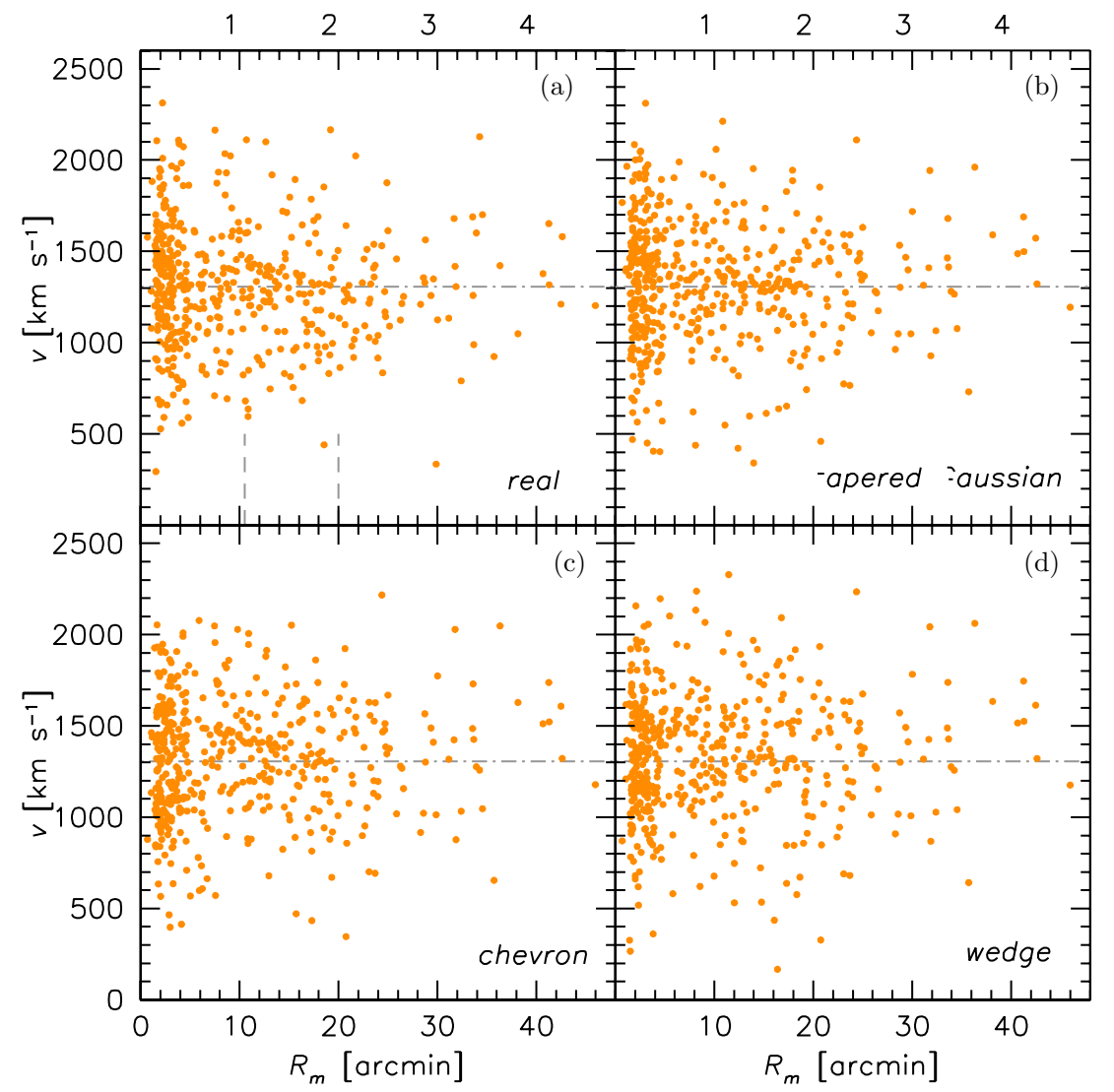

Figure 7. Simple models for GC substructure in phase space. (a) The real data. Vertical dashed lines show the radial range where the models are fitted. (b) Mock data set using a tapered double-Gaussian model. (c) Mock data set using a Gaussian+chevron model. (d) Mock data set using a Gaussian+wedge model. Some single-Gaussian models can also be seen in Figures 5(b) and (c) for comparison. The real data appear to have a phase-space morphology intermediate to these various two-component models, but closest to the chevron-based model.

(A color version of this figure is available in the online journal.)

where $v^{\prime}$ is the slope and $R_{\mathrm{x}}$ is the apex radius. The systemic velocity is set as a fixed parameter $v_{\text {sys }}=1307 \mathrm{~km} \mathrm{~s}^{-1}$.

We fit the various model alternatives to the shell region and find $f_{\mathrm{s}} \sim 0.22,0.23$, and 0.26 for the tapered-Gaussian, chevron, and wedge models, respectively. Examples of mock data generated from the best model fits are shown in Figure 7.
By eye, the real data suggest a feature that is intermediate between the different models, but most closely related to the chevron, owing to the strong "edge" seen in the substructure. More quantitatively, Monte Carlo modeling of likelihood fits is unfortunately not very informative about which model is the best representation of the substructure. We find that any of the 


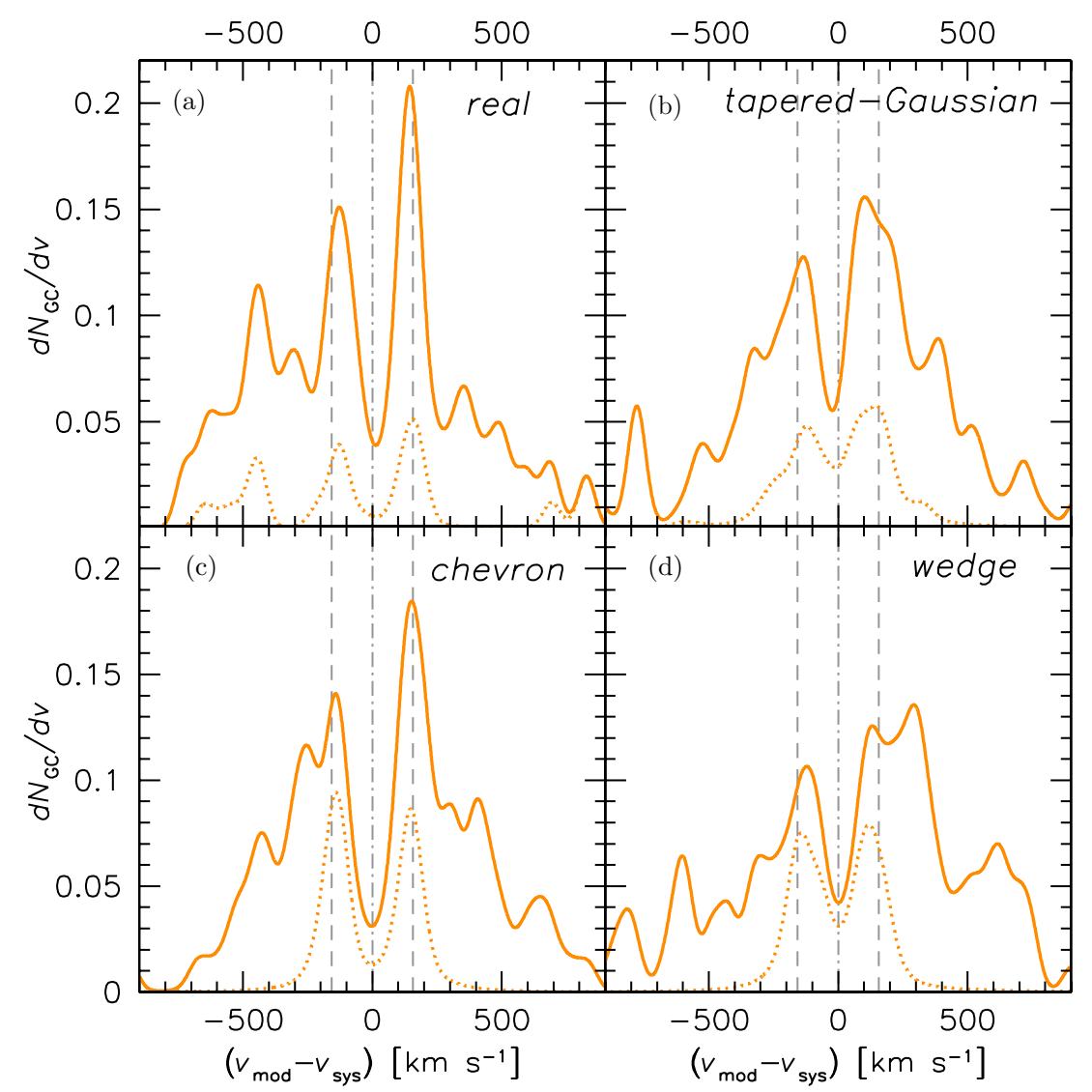

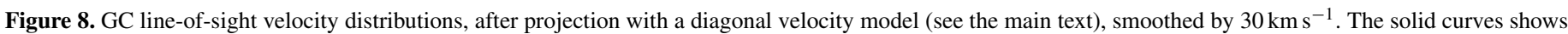

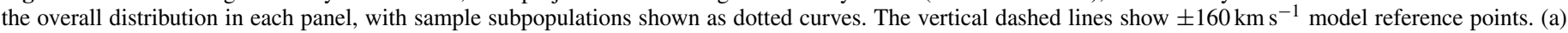

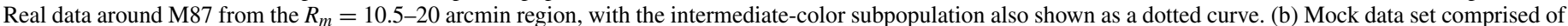

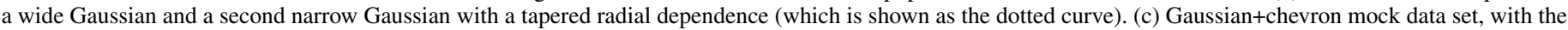

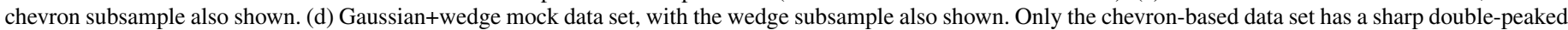
velocity distribution as in the real data, after projecting with the diagonal model.

(A color version of this figure is available in the online journal.)

alternative models can readily fit mock data based on the other models.

Instead, we turn to a somewhat less parametric representation of the data, using simply a diagonal model (with a characteristic slope $v^{\prime}=17.3 \mathrm{~km} \mathrm{~s}^{-1} \operatorname{arcmin}^{-1}$ ) to project each data point to a velocity at a radius of $R_{m}=10 \mathrm{arcmin}$. This exercise allows us to collapse all of the position-velocity phase-space data into single one-dimensional LOSVDs analogous to Figure 6, comparing real data to simple models.

The results are shown in Figure 8 , where a $30 \mathrm{~km} \mathrm{~s}^{-1}$ Gaussian smoothing kernel has been applied in order to capture the shape of the distribution while reducing Poisson noise. Given an apex in all cases of $R_{\mathrm{x}} \simeq 19$ arcmin, the model projects to characteristic velocities of $v_{\text {mod }} \sim 160 \mathrm{~km} \mathrm{~s}^{-1}$ (relative to $v_{\text {sys }}$ ) at 10 arcmin. Applying the projection to mock data, two peaks are always generated but other properties are more model dependent: the peak widths, locations relative to $v_{\text {mod }}$, and dips in between. In particular, a chevron model when added to a Gaussian produces the most distinct, narrow peaks close to $v_{\text {mod }}$.

We find by inspection of Figure 8 that the real data most closely resemble the chevron-based model, with narrow, welldefined peaks near $v_{\text {mod }}$. This plot also indicates a two-sided rather than one-sided chevron (see also Figure 6). There is, furthermore, an additional narrow feature near $-400 \mathrm{~km} \mathrm{~s}^{-1}$ that appears stronger than a random fluctuation and suggests the presence of another sharp edge in phase space terminating at $R_{m} \sim 30$ arcmin (see also Figures 3(b) and 6), i.e., perhaps tracing a second, fainter shell.

Although our overall conclusion from LOSVD analyses is that a chevron is the best representation for the shell, it is difficult to be certain given the available data and the uncertainties in the coordinate transformations to $R_{m}$. The real shell probably has a morphology somewhat intermediate between the idealized models studied here, and also shows hints of being asymmetric with respect to $v_{\text {sys }}$. For now, we report the best-fit parameters for the favored chevron model, with uncertainties based on Monte Carlo simulations: $f_{\mathrm{s}}=0.23 \pm 0.06, \sigma_{1}=19 \pm 10 \mathrm{~km} \mathrm{~s}^{-1}$, $v^{\prime}=15.2 \pm 1.5 \mathrm{~km} \mathrm{~s}^{-1} \operatorname{arcmin}^{-1}$, and $\sigma_{2}=358 \pm 27 \mathrm{~km} \mathrm{~s}^{-1}$. Of the 119 confirmed GCs in the $R_{m}=50-95 \mathrm{kpc}$ region, we thus estimate that $21-35$ of them are in the shell. We highlight the 27 most likely shell members based on this chevron model in Figure 1(b).

Note that the quoted error bars on these fit parameters are the statistical uncertainties for an assumed simple chevron model. The systematic uncertainties from considering alternative models, and from allowing for different radius conventions (Section 3.1), are difficult to quantify. Above, we also reported a maximal-ignorance model that disregards any spatial tapering of the substructure by fitting a simple double-Gaussian, and returns a very conservative upper limit of $120 \mathrm{~km} \mathrm{~s}^{-1}$ on the dispersion. 

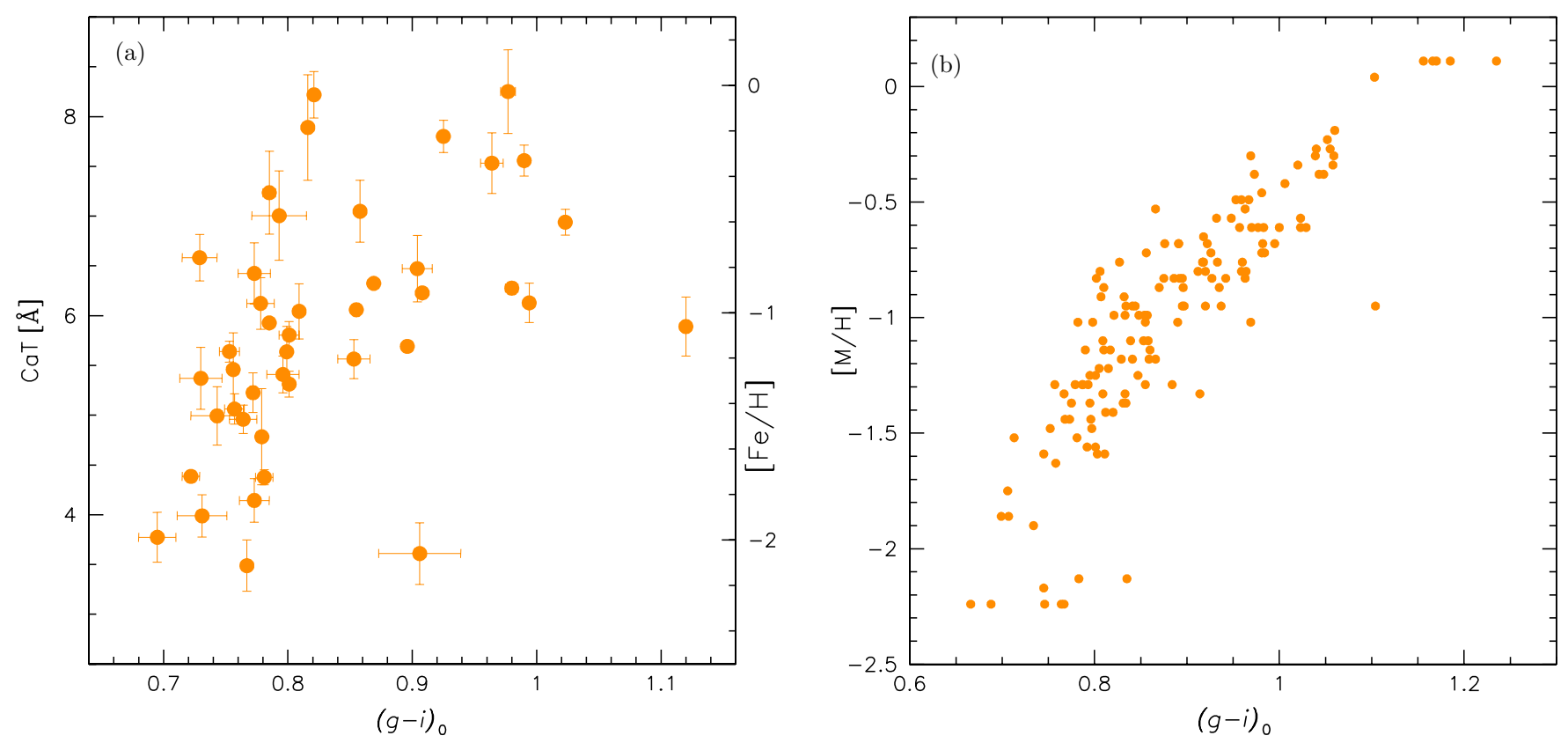

Figure 9. Color vs. metallicity for M87 GCs. (a) Results from CaT analysis of the subset of our DEIMOS spectra with good enough signal-to-noise (S/N $\gtrsim 12$ ) and with $(g-i)_{0}$ data available (44 objects). The left axis shows the direct CaT line strengths, where the error bars indicate $68 \%$ uncertainties. The right axis shows the equivalent iron metallicity. The color uncertainties in some cases are smaller than the point sizes. One additional outlier is off the scale at $(g-i)_{0}=0.91$, CaT $=$ 1.9 Å. (b) Color vs. metallicity for $148 \mathrm{GCs}$ from earlier spectroscopic analysis (Cohen et al. 1998). Note that while in this case the line-index metallicity conversions below $[\mathrm{M} / \mathrm{H}] \sim-1.8$ may well be nonlinear, and above $[\mathrm{M} / \mathrm{H}] \sim 0$ the metallicities are not well calibrated, the important issue here is not the exact metallicities, but rather the monotonicity of the color-metallicity relation, which is demonstrated by both data sets.

(A color version of this figure is available in the online journal.)

\subsection{Color and Metallicity Distributions}

One great advantage of using GCs as phase-space tracers is that they provide additional measurable properties beyond position and velocity. Because each GC is to a good approximation comprised of a homogeneous stellar population with a single age and metallicity, the principles of stellar-subpopulation tagging used in the Milky Way (Helmi 2008) can be extended to more distant systems. In particular, GCs are now known to be generally very old objects (ages of $\sim 10 \mathrm{Gyr}$ ), both in M87 (Cohen et al. 1998) and in other galaxies (Brodie \& Strader 2006). Therefore, their optical colors are dominated by metallicity variations rather than by ages, and we can use color as a good metallicity proxy.

Rather than solely relying on literature findings on this point, we consider the color-metallicity link in our own data set. From the DEIMOS spectra, there are 47 objects with high enough signal-to-noise ratios $(\mathrm{S} / \mathrm{N})$ to estimate iron-based metallicities $[\mathrm{Fe} / \mathrm{H}]$ via the $\mathrm{CaT}$ line strengths, using techniques that we have developed earlier with the same instrumental setup (Foster et al. 2010, 2011). Approximate line-strength uncertainties are estimated from the spectroscopic $\mathrm{S} / \mathrm{N}$, after calibration from Monte Carlo simulated observations of model spectra.

We provide these metallicity estimates in Table 2, and in Figure 9(a) plot them versus dereddened colors for the same GCs where $(g-i)_{0}$ data are available. We find a strong correlation between metallicity and color that agrees roughly with model predictions (Vazdekis et al. 2003). Understanding the scatter in the data will require more detailed investigation.

This test does not prove unequivocally that these colors are a perfect proxy for metallicity, given various unresolved issues about CaT-based metallicities, and the possibility of some residual age effects on the colors. However, it does provide assurance that the current conventional wisdom about GC color as a reliable metallicity proxy is probably sound in the halo of M87 (i.e., at radii of $\sim 45-220 \mathrm{kpc}$ ).

Previous spectroscopic work using bluer wavelengths at smaller radii $(\sim 3-40 \mathrm{kpc})$ also found M87 GC metallicity indices to correlate strongly with $U-R$ color, and for the ages to be almost all larger than $10 \mathrm{Gyr}$ (Cohen et al. 1998). We revisit these data by comparing our $(g-i)_{0}$ colors to these authors' metallicity estimates in Figure $9(\mathrm{~b})$, finding a tight correlation between the two quantities. Again, this supports the color-metallicity link.

Despite these arguments that color is a good proxy for metallicity in our data set, it is important to note that our analysis below is independent of this link. Whatever the root cause of variations in color, we can still use it as an empirical tagging factor. Then for our current purposes, the issue is more a matter of semantics: whether we are analyzing chemodynamics or chromodynamics.

Our first basic goal is to use color as a completely independent dimension in the data, to see if it supports the differences among GCs that emerged from the position-velocity phasespace analyses. We plot velocity versus color in the shell region in Figure 10(a). In M87, as in galaxies in general, the population of metal-poor GCs has a more extended distribution from their host galaxy's center than the more metal-rich GCs. Given that all of these objects reside in the same gravitational potential, and assuming that they have similar orbital anisotropies, their spatial differences are expected to be related to their velocity dispersions. The metal-rich GCs should generally have lower dispersions, and Figure 10(a) is expected to show a systematic decrease in dispersion with color, with perhaps a strong transition between the two main subpopulations, at $(g-i)_{0} \sim 0.9$. 
Table 2

CaT Measurements of M87 Globular Clusters

\begin{tabular}{|c|c|c|c|c|c|}
\hline ID & $\begin{array}{c}\text { R.A. } \\
\text { (J2000) }\end{array}$ & $\begin{array}{c}\text { Decl. } \\
(\mathrm{J} 2000)\end{array}$ & $i_{0}$ & $(g-i)_{0}$ & $\begin{array}{l}\mathrm{CaT} \\
(\AA)\end{array}$ \\
\hline 731 & 187.72452 & 12.28682 & 20.37 & $0.858 \pm 0.004$ & $7.05 \pm 0.31$ \\
\hline 878 & 7.70708 & 12.28149 & 20.62 & $.816 \pm 0.004$ & $7.89 \pm 0.53$ \\
\hline S952 & 187.69952 & 12.27727 & 20.58 & $0.779 \pm 0.004$ & $4.78 \pm 0.48$ \\
\hline S1007 & 187.69344 & 12.28971 & 19.41 & $1.023 \pm 0.002$ & $6.94 \pm 0.13$ \\
\hline 1074 & 187.68856 & 12.27776 & 20.29 & $1.120 \pm 0.004$ & $5.89 \pm 0.30$ \\
\hline 1265 & 187.67009 & 12.28303 & 19.82 & $0.772 \pm 0.003$ & $5.23 \pm 0.20$ \\
\hline H20493 & 187.87314 & 12.15391 & 20.13 & $0.785 \pm 0.005$ & $7.24 \pm 0.42$ \\
\hline H21863 & 187.87253 & 12.17069 & 20.54 & $0.977 \pm 0.006$ & $8.25 \pm 0.42$ \\
\hline H25785 & 187.86159 & 12.21268 & & $0.914 \pm 0.002$ & $1.95 \pm 0.22$ \\
\hline H26690 & 187.72956 & 12.22270 & 19.70 & $0.990 \pm 0.002$ & $7.56 \pm 0.16$ \\
\hline H27496 & 187.80753 & 12.23123 & 19.56 & $0.801 \pm 0.002$ & $5.31 \pm 0.13$ \\
\hline H27916 & 187.71521 & 12.23610 & 20.45 & $0.756 \pm 0.004$ & $5.46 \pm 0.37$ \\
\hline H28411 & 187.70221 & 12.24179 & 20.10 & $0.799 \pm 0.002$ & $5.64 \pm 0.26$ \\
\hline H28415 & 187.78343 & 12.24165 & 19.71 & $0.925 \pm 0.002$ & $7.80 \pm 0.16$ \\
\hline H28866 & 187.75563 & 12.24666 & 19.96 & $4 \pm 0.004$ & $6.13 \pm 0.20$ \\
\hline Н30757 & 187.7 & 12.26691 & 20.20 & \pm 0.002 & $6.04 \pm 0.28$ \\
\hline Н30772 & 187.7 & 12.26728 & 19.84 & $=0.033$ & $3.61 \pm 0.31$ \\
\hline Н31134 & 187.72735 & 12.27091 & 20. & $0.821 \pm 0.003$ & $8.22 \pm 0.23$ \\
\hline T13456 & 187.92093 & 12.46766 & 21.14 & $7 \pm 0.008$ & $5.06 \pm 0.15$ \\
\hline $\mathrm{T} 13500$ & 187.93052 & 12.42406 & 20.55 & $7 \pm 0 .($ & $3.49 \pm 0.26$ \\
\hline T13559 & 187.93331 & 12.37800 & 21.87 & 0.9 & $6.47 \pm 0.33$ \\
\hline T13569 & 187.98 & 12.36469 & 21. & 0.6 & $3.77 \pm 0.25$ \\
\hline $\mathrm{T} 13571$ & 187.89063 & 12.36288 & & 0.8 & $5.81 \pm 0.14$ \\
\hline & & & & & $4.14 \pm 0.22$ \\
\hline T13593 & 187.90345 & 12.35 & 21. & $=0.011$ & $6.12 \pm 0.26$ \\
\hline $\mathrm{T} 13609$ & 187.96307 & 12.34319 & 21.95 & $0.729 \pm 0.014$ & $6.58 \pm 0.23$ \\
\hline T13611 & 187.92010 & 12.34146 & 22.44 & $3 \pm 0.022$ & $7.00 \pm 0.45$ \\
\hline T13623 & 187.90570 & 12.33556 & 21.49 & $0.764 \pm 0.011$ & $4.96 \pm 0.14$ \\
\hline T13642 & 187.94181 & 12.32521 & 20.39 & \pm 0.004 & $6.32 \pm 0.02$ \\
\hline T13648 & 187.98298 & 12.323 & & $1 \pm 0.020$ & $3.99 \pm 0.21$ \\
\hline T13899 & 187.84033 & 12.42620 & & $0.964 \pm 0.009$ & $7.53 \pm 0.31$ \\
\hline $\mathrm{T} 13901$ & 187.87673 & 12.42616 & 21. & \pm 0. & $5.56 \pm 0.20$ \\
\hline T14060 & 187.87089 & 12.38884 & 20.83 & $0.722 \pm 0.007$ & $4.39 \pm 0.06$ \\
\hline T14108 & 187.83846 & 12.37504 & 19.82 & $8 \pm 0.002$ & $6.23 \pm 0.01$ \\
\hline T14133 & 187.84645 & 12.37052 & 20.69 & $0.980 \pm 0.005$ & $6.28 \pm 0.06$ \\
\hline T14149 & 187.87374 & 12.36463 & & $0.773 \pm 0.013$ & $6.42 \pm 0.31$ \\
\hline T14228 & 187.84434 & 12.35020 & 20.44 & $0.785 \pm 0.004$ & $5.93 \pm 0.03$ \\
\hline $\mathrm{T} 15685$ & 188.04605 & 12.36726 & 22.08 & $0.730 \pm 0.017$ & $5.37 \pm 0.31$ \\
\hline $\mathrm{T} 15777$ & 188.14226 & 12.45638 & 21.93 & $0.796 \pm 0.013$ & $5.41 \pm 0.19$ \\
\hline T15863 & 188.17885 & 12.36688 & 21.36 & $0.753 \pm 0.008$ & $5.64 \pm 0.11$ \\
\hline T15886 & 188.15205 & 12.34920 & 22.33 & $0.743 \pm 0.021$ & $4.99 \pm 0.29$ \\
\hline T15900 & 188.21484 & 12.33844 & 20.6 & & $4.20 \pm 0.06$ \\
\hline T15908 & 188.15419 & 12.33126 & 21.19 & $0.781 \pm 0.007$ & $4.38 \pm 0.08$ \\
\hline T16080 & 188.27954 & 12.34479 & 21.0 & & $6.21 \pm 0.07$ \\
\hline $\mathrm{T} 17211$ & 188.33727 & 12.48077 & 21.0 & $\ldots$ & $5.08 \pm 0.08$ \\
\hline VUCD6 & 187.86816 & 12.41766 & 18.63 & $0.896 \pm 0.001$ & $5.69 \pm 0.01$ \\
\hline VUCD8 & 188.01813 & 12.34176 & 18.96 & $0.855 \pm 0.001$ & $6.06 \pm 0.01$ \\
\hline
\end{tabular}

Notes. Note that the $(g-i)_{0}$ color uncertainties are based on shot noise only and are useful for relative comparisons of colors in this data set. The absolute uncertainties from calibration, reddening correction, background estimation, etc., will be larger. The equivalent iron metallicities shown on the right-hand axis of Figure 9(a) are derived using the expression: $[\mathrm{Fe} / \mathrm{H}] \quad 0.438 \times \mathrm{CaT}-3.641$.

(This table is also available in a machine-readable form in the online journal.)

Figure 10(a) does show a kinematic transition with color, but not in the way just described. There is an overall pattern of a cold component near $v_{\text {sys }}$ embedded in a hotter component (cf. Figure 6), but the cold component does not appear blueward of $(g-i)_{0} \sim 0.77$. This color is in the middle of the main blue peak for M87's GC system, and implies that there is kinematical substructure within this peak. ${ }^{9}$ We have looked at the same

\footnotetext{
9 This is again a test that is insensitive to the radius transformation $R_{m}$.
}

information using the CaT-based metallicities, with consistent results (colder material at $\mathrm{CaT} \gtrsim 5.3 \AA$ or $[\mathrm{Fe} / \mathrm{H}] \gtrsim-1.3 \mathrm{dex}$ ), but the statistics are too poor to be conclusive (17 GCs in the shell region).

We next take a different approach, of separating GCs into probable shell and non-shell objects, based on the phase-space maximum-likelihood models constructed previously. We then examine the color distributions of the two subsamples. For each of the models (tapered Gaussian, chevron, and wedge), the two color distributions are different, with the chevron case shown in Figure 10(b) as an example. ${ }^{10}$ Here the shell appears to contain an excess population of intermediate-color GCs, peaking at $(g-i)_{0} \sim 0.90$.

Motivated by this result, we also show the velocity distribution of all the intermediate color objects in the shell region, with $(g-i)_{0}=0.85-0.95$, in Figure 8(a). These objects do show a double-peaked profile as expected in the chevron model, except for an additional low-velocity peak that accounts for much of the candidate outer shell. We have also fitted the chevron model to the phase-space data for the blue, intermediate-color, and red GCs separately. The resulting parameters for each of these fits are mutually consistent, and color does not discriminate sharply between shell and non-shell objects, but we do find that the shell fraction is highest in the intermediate-color population, with $f_{\mathrm{s}} \sim 0.5$.

We evaluate the statistical significance of the differences between the chevron and non-chevron color distributions using a Kolmogorov-Smirnov (K-S) test. We find a $D$ value of 0.25 , with a $13 \%$ probability of occurrence by chance. According to the test, the largest difference between the distributions comes at the far blue end, supporting the transition seen in Figure 10(a). The same is true for all three shell models, with the far blue difference being strongest for the tapered-Gaussian case, and the intermediate-color peak for the chevron case. The similarity of the three models also suggests that these conclusions are insensitive to the choice for radius $R_{m}$, as is also explicitly the case for the test in Figure 10(a).

We have found that metallicity information (primarily using color as a proxy) provides independent support for the presence of the M87 shell. The colors do not yet differentiate clearly between the alternative phase-space models for the shell, but even our unprecedented data set only scratches the surface of what can now be accomplished. It is technically feasible to increase the number of measured GC velocities by an order of magnitude, e.g., to $\sim 1500$ objects in the shell region alone.

\subsection{Progenitor Inferences from GCs}

Having established the existence of the shell, and some of its properties, we now attempt to identify the nature of its progenitor, assuming that the accretion and disruption of a single galaxy was responsible. Unfortunately, we will see that it is difficult to derive a self-consistent solution from the basic shell parameters, so we will carry out an inventory of different types of constraints to see if an overall solution emerges (with an estimate of the progenitor's $V$-band stellar luminosity, $L_{V}$, as a basic goal).

Since in the previous section we have just discussed the color distribution of the shell GCs, it is natural to try using these colors to constrain the progenitor $L_{V}$. Empirically, GC colors correlate with host galaxy luminosity (e.g., Larsen et al. 2001; Strader

\footnotetext{
10 Among our probable shell sample, there are two ultracompact dwarf candidates: VUCD1 and H59823.
} 

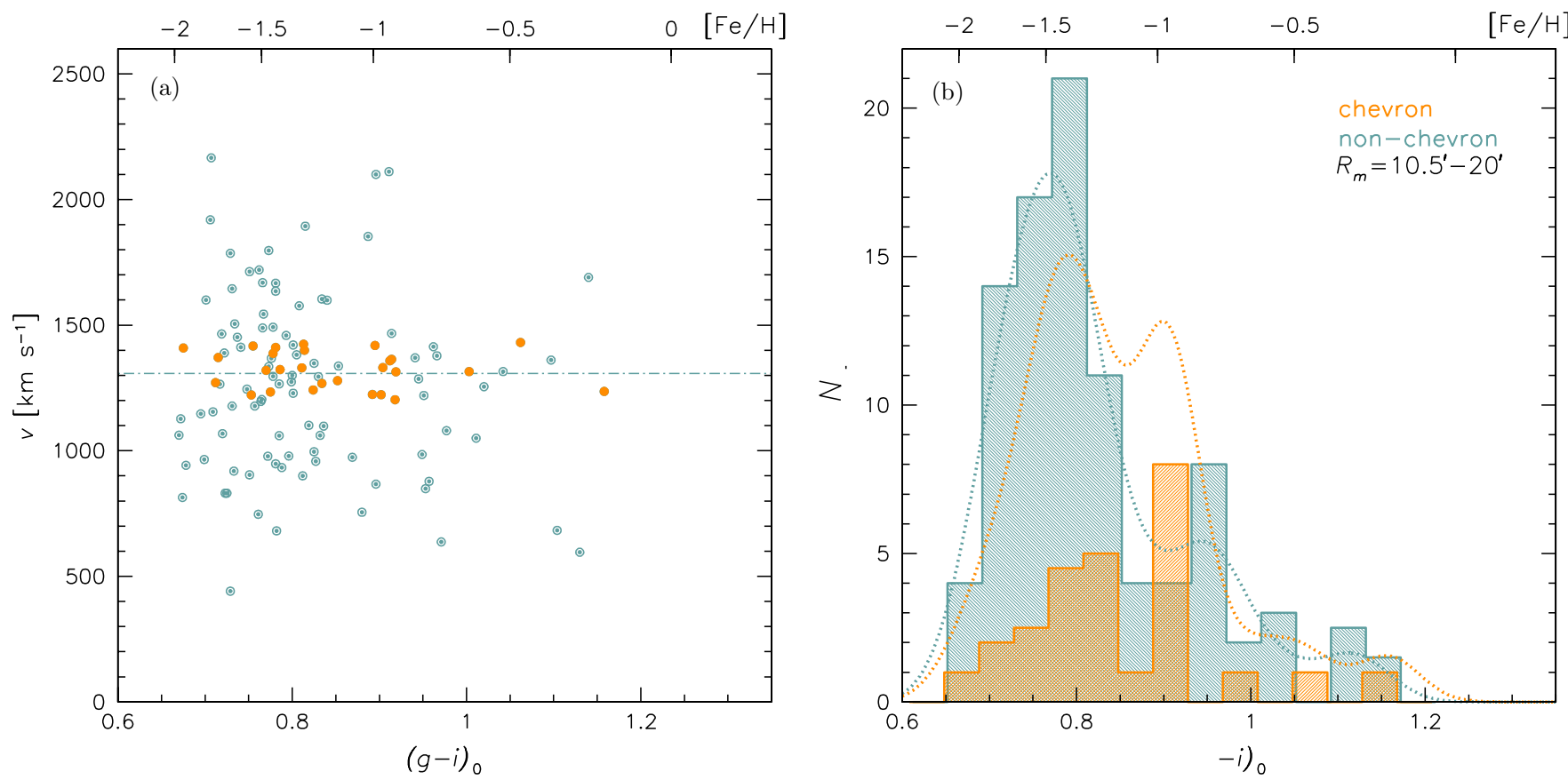

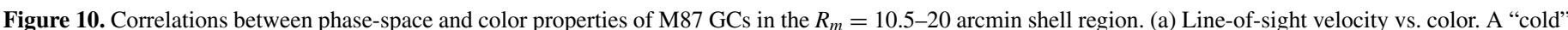

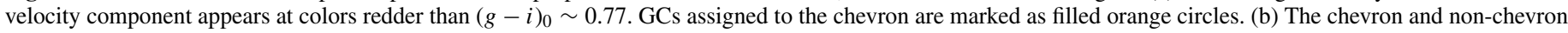

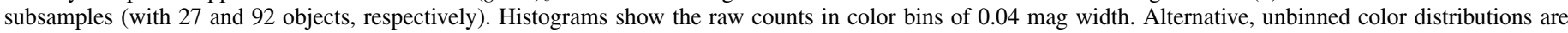

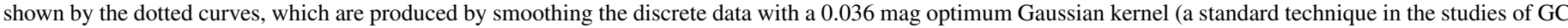

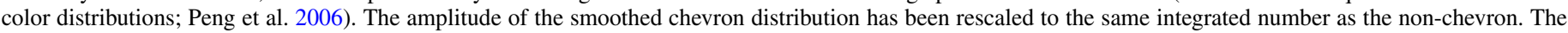
chevron GCs appear to have a slightly redder "blue peak" than the non-chevron GCs, as well as a strong intermediate-color peak.

(A color version of this figure is available in the online journal.)

et al. 2004; Lotz et al. 2004), which is conceptually related to the correlations between substructure metallicities and their progenitor masses (e.g., Johnston et al. 2008). This framework was used to estimate the relative masses of accreted galaxies in the halo of a nearby S0 in Arnold et al. (2011).

In the case of M87, we can make use of the correlations found from the "ACSVCS" large survey of elliptical and lenticular galaxies, both giant and dwarf, in the Virgo cluster (Peng et al. 2006). Although the GC color distributions in these galaxies are generally complex, they show a general tendency for bimodality, with distinct blue and red peaks. The color-luminosity relation is therefore reported separately for the blue and red GCs. The underlying basis for these correlations is thought to be a mass-metallicity relation, but again, our analysis does not rely on this conclusion.

We have modeled the color distributions of the chevron and non-chevron GCs (Figure 10(b)) using Kaye's Mixture Models based on heteroscedastic Gaussian color subpopulations (Ashman et al. 1994). We find marginal significance ( $p=$ $0.325)$ for bimodality among the chevron GCs, and high significance $(p<0.001)$ for the non-chevron GCs. Beginning with the non-chevron GCs, we find that they have a primary peak at $(g-i)_{0} \sim 0.77$ and a possible weak secondary peak at $(g-i)_{0} \sim 0.94$, which correspond to $(g-z)_{0} \sim 0.92$ and $\sim 1.17$, or $[\mathrm{Fe} / \mathrm{H}] \sim-1.5$ and -0.8 , respectively (where we have made empirical color conversions using a large set of M87 GCs that overlap between Advanced Camera for Surveys and CFHT imaging).

We next assume for simplicity that the GC color peaks in the surviving Virgo early-type galaxies are representative of the disrupted progenitors that built up the M87 halo. ${ }^{11}$

\footnotetext{
11 There is evidence that galaxies accreted during earlier epochs had systematically redder GC colors at a fixed mass (Brodie \& Strader 2006),
}

Considering first the peak locations of the non-chevron GCs and comparing them to the non-parametric peaks determined for the ACSVCS galaxies (Peng et al. 2006), we find the former to be fairly atypical when considered jointly (a puzzle that would be worsened if the chevron GCs were included). This makes the progenitor inference unclear, perhaps owing to the superposition of color peaks from multiple progenitors, but our best guess would be a typical progenitor magnitude of $M_{V} \sim-18\left[\log \left(L_{V} / L_{V, \odot}\right) \sim 9.5\right]$.

Approximating the $V$-band stellar mass-to-light ratios as roughly constant, the implication would be that the accretion events which built up the bulk of the M87 halo had characteristic stellar-mass merger ratios of $\sim 1: 30$. This may be consistent with current ideas of massive galaxies' stellar envelopes being assembled through minor mergers (e.g., Oser et al. 2012).

Turning now to the chevron GCs, they have a primary peak at $(g-i)_{0} \sim 0.79$ and a secondary peak at $(g-i)_{0} \sim 0.90$, corresponding to $(g-z)_{0} \sim 0.95$ and $\sim 1.11,[\mathrm{Fe} / \mathrm{H}] \sim-1.4$ and -1.0 . This red peak location is very atypical for the ACSVCS sample and may have been inaccurately estimated through our procedure of statistically disentangling the chevron and nonchevron GCs. We therefore consider the color constraint on the shell progenitor luminosity to be indeterminate.

The other two major clues about the shell origin are its number of GCs, $N_{\mathrm{GC}}$, and their mutual velocity dispersion, $\sigma_{\mathrm{GC}}$. For both of these properties, we use the results of maximum-likelihood models from Section 3.4. While our default constraint comes from the "chevron" model, we will also account for the systematic uncertainty in the shell morphology (e.g., owing to the radius conventions discussed in Section 3.1) by considering an alternative scenario of maximal ignorance of the morphology. For

which would mean that our luminosities for the typical M87 halo progenitors will be overestimated. 


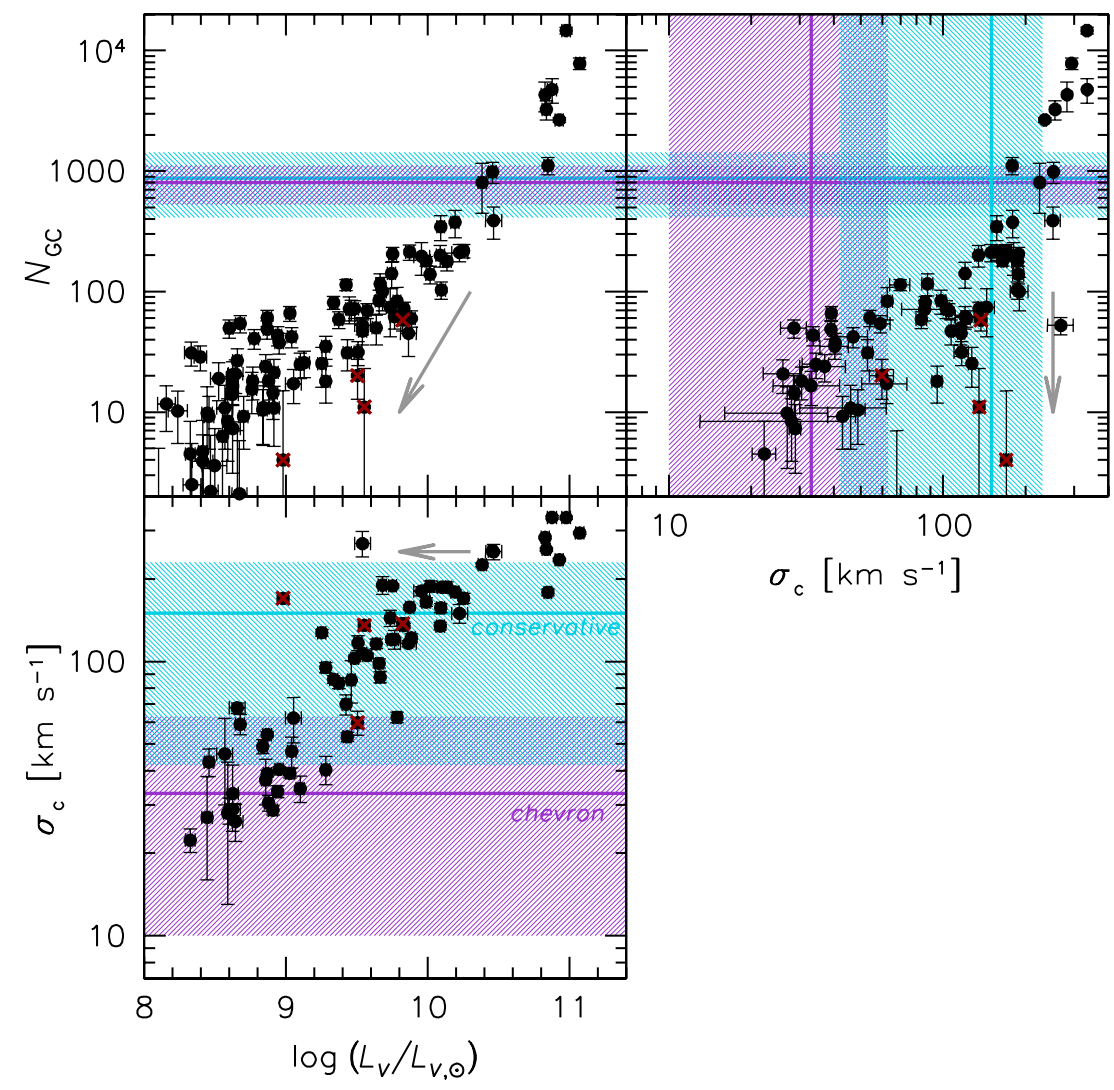

Figure 11. Parameter space of candidate progenitor galaxies for the M87 shell, plotting $V$-band luminosity, central stellar velocity dispersion, and GC numbers. The bands show constraints from modeling the shell GCs, with the narrower purple bands stemming from our default chevron-based analysis, and the wider blue bands showing a model that does not incorporate spatial information (yielding a conservative upper limit on the dispersion). The points with error bars show early-type galaxies in Virgo from the ACSVCS survey, with $N_{\mathrm{GC}}$ and $L_{V}$ from Peng et al. (2008). The dispersions are mostly from HyperCat with some additions from Chilingarian (2009) and Toloba et al. (2011). Points with red $\times$ symbols superimposed show four low-luminosity ellipticals near to M87, whose positions in these diagrams are suggestive of histories of stripping - as illustrated schematically by the gray arrows. The constraints from the chevron model do not match up well with any realistic candidate progenitor galaxies, while the conservative model is more viable (see the text for details).

(A color version of this figure is available in the online journal.)

this case we adopt the double-Gaussian analysis of Section 3.4, which leads to a much larger value of $\sigma_{\mathrm{GC}}\left(50-120 \mathrm{~km} \mathrm{~s}^{-1}\right.$ rather than $10-30 \mathrm{~km} \mathrm{~s}^{-1}$ ).

To make use of the $N_{\mathrm{GC}}$ and $\sigma_{\mathrm{GC}}$ constraints, we must correct both observational measurements to the relevant quantities for the progenitor galaxies. In the case of $N_{\mathrm{GC}}$, we extrapolate the fraction of shell GCs in our spectroscopic sample to the much larger sample of unobserved GCs in the same region. We have constructed a smooth model surface density profile of the M87 GC system, based on both the CFHT photometric data as well as spectroscopic data to confirm the non-GC contamination rate (see $\mathrm{S}+11)$. This model is initially based on our photometric limit of $i=22.5$ ( $z \quad 22.4)$, but to get total GC numbers we need to extrapolate to fainter luminosities, using an evolved Schechter function fitted to the central GCs of M87 (Jordán et al. 2007). This provides a scaling factor of $2.76 .{ }^{12}$

Now, integrating the revised surface density between 10 and 20 arcmin where the shell signal is clear, we find a total of $3500 \pm 400 \mathrm{GCs}$. Multiplying by the inferred $f_{\mathrm{s}}$ values for both the chevron and double-Gaussian models (see Section 3.4), we estimate $N_{\mathrm{GC}}=800_{-270}^{+320}$ and $870_{-460}^{+560}$ total GCs in the shell, respectively (integrating over all magnitudes). These numbers could be lower if we have detected a disproportionate fraction of the shell objects by spatial bias in our spectroscopic selection

\footnotetext{
12 With a Gaussian luminosity function, this factor would be 2.50 , but for direct comparison with $N_{\mathrm{GC}}$ values from the ACSVCS, we adopt 2.76 .
}

that coincides with a spatial clumping of the shell; or higher if the shell extends farther in radius and velocity than in our simple model (see, e.g., Figure 2(b)). It is difficult to quantify such uncertainties, but we suspect that they could involve an additional factor of $\sim 2$, which would accommodate a range of up to $\sim 200-2900$ GCs. If $N_{\mathrm{GC}} \sim 800$, then the shell accounts for $\sim 20 \%$ of the GCs around M87 within the $50-100 \mathrm{kpc}$ range and $\sim 5 \%$ of the total GC system. ${ }^{13}$

What kinds of galaxies are observed to host so many GCs? To provide a visual overview of this constraint, we plot the number of GCs versus host luminosity for the ACSVCS sample in the upper left panel of Figure 11, finding that $\sim L^{*}$ luminosities of $\log \left(L_{V} / L_{V, \odot}\right) \sim 10.1-10.8$ are implied.

Next we consider the shell's internal velocity dispersion, which as a first approximation, we assume to be similar to the internal velocity dispersion of its progenitor, as might be expected under the simplest tidal stripping models. Here the comparison to the ACSVCS galaxies is less straightforward, since very few of these have direct measurements available of their GC velocity dispersions. Instead, we will adopt a

\footnotetext{
13 To derive the latter value, we have integrated the GC spatial density profile out to a radius of $45 \operatorname{arcmin}(220 \mathrm{kpc})$, which is where the planetary nebula (PN) kinematics suggest a transition between objects bound and not bound to M87 (Doherty et al. 2009; the kinematics data for the GCs themselves are less extensive at large radii and do not yet show any sign of such a transition). The total GC population is then $\sim 16,200$.
} 
conversion between $\sigma_{\mathrm{GC}}$ and the widely available central stellar velocity dispersion $\sigma_{\mathrm{c}}$. Based on the forthcoming kinematical analysis of a handful of low-luminosity E/SOs (V. Pota et al., in preparation), we find that the ratio $\sigma_{\mathrm{c}} / \sigma_{\mathrm{GC}}$ varies from galaxy to galaxy over a range $\sim 1.3-2.3$, with a typical value of $\sim 1.8$. We therefore infer $\sigma_{\mathrm{c}} \sim 35 \pm 25 \mathrm{~km} \mathrm{~s}^{-1}$ and $\sim 150 \pm 100 \mathrm{~km} \mathrm{~s}^{-1}$ based on the chevron and double-Gaussian models, respectively.

We plot this constraint again versus the ACSVCS sample in the bottom left panel of Figure 11. Here we see that the chevron-based velocity dispersion suggests a dwarf E/S0 with $\log \left(L_{V} / L_{V, \odot}\right) \lesssim 9.5,{ }^{14}$ while the conservative upper-limit dispersion allows for luminosities of up to $\log \left(L_{V} / L_{V, \odot}\right) \sim$ 10.6 .

For the chevron model, the dispersion constraints on luminosity are thus inconsistent with the constraints from GC numbers. To make this comparison more directly, we show dispersion versus GC numbers in the upper right panel of Figure 11. The intersections of the vertical and horizontal bands mark out possible solutions for the progenitor based on the shell analyses-but these do not overlap with the properties of known Virgo galaxies. It does not seem plausible that either a dwarf galaxy hosted hundreds of GCs, or that an $\sim L^{*}$ galaxy left extensive debris with a velocity dispersion of $\lesssim 30 \mathrm{~km} \mathrm{~s}^{-1}$.

The conservative model fares much better in this respect, as several ACSVCS galaxies are consistent with both its $N_{\mathrm{GC}}$ and $\sigma_{\mathrm{GC}}$ constraints. Some examples include NGC 4435 and NGC 4473, with $\log \left(L_{V} / L_{V, \odot}\right) \sim 10.1-10.2$. This model seems promising, but one should remember that it is intended to represent a bracketing case of maximal ignorance about the shell morphology, while there does seem to be some degree of substructure tapering with radius in phase space, independently of the radius convention. Therefore, it is worthwhile reviewing any additional lines of evidence about the accretion progenitor.

If the progenitor was very massive, then it should have left other clear signatures of its passing, besides the phasespace shell. One might expect to see obvious disturbances and asymmetries in the light distribution of M87, as well as strong merger-induced halo rotation, which is not observed $(S+11)$. If we assume that this rules out a mass ratio of $\sim 1: 3$ or higher, it implies $\log \left(L_{V} / L_{V, \odot}\right) \lesssim 10.4$ for the progenitor, or perhaps somewhat higher if dark matter is taken into account.

Besides the impact of the accretion event on M87, the incoming galaxy should have deposited not only GCs but also field stars, which would leave a large-scale surface brightness fluctuation across the face of M87. In the shell region where the typical surface brightness is $\mu_{V} \sim 27 \mathrm{mag} \mathrm{arcsec}^{-2}$, we can rule out obvious fluctuations at the $\sim 0.5 \mathrm{mag} \operatorname{arcsec}^{-2}$ level (see Figure 1(a) and Janowiecki et al. 2010). We can therefore rule out $\log \left(L_{V} / L_{V, \odot}\right) \gtrsim 9.8$ of accreted stars that are distributed uniformly over the shell region. ${ }^{15}$

\footnotetext{
14 An alternative mechanism for producing a low velocity dispersion is through tidal tails stripped out from the cold disk of an infalling spiral galaxy (e.g., Hernquist \& Spergel 1992; Hibbard \& Mihos 1995). However, we do not know of any galaxy with hundreds of GCs contained in a cold disk. Alternatively, some GCs could have been formed from cold gas in such a merger, and then deposited on closely associated orbits. Because of the age-metallicity degeneracy, the shell GCs might in principle be young and metal-rich rather than old and metal-poor. Full analysis of the GC ages is beyond the scope of this paper, but our initial inspection of the Hectospec data indicates there are no objects as young as $\sim 1$ Gyr.

15 This constraint is relevant not only to our default single-progenitor scenario, but also to a scenario involving multiple small galaxies that together deposited a large number of GCs with cold kinematics, e.g., from $\sim 5$ dwarf E/S0s with $\log \left(L_{V} / L_{V, \odot}\right) \sim 9.3$ each. Besides the low probability of accreting such galaxies on near-identical trajectories, the associated stellar debris could violate the surface brightnesses constraint.
}

This luminosity limit is lower if the stars are confined to only one side of M87, as Figure 1(a) suggests; and higher if the stars have become spatially decoupled from the GCs and reside at smaller radii where they are either hard to detect, or are still intact as one of the nearby low-luminosity elliptical galaxies. Such spatial segregation is plausible at some level since the GCs would be expected to preferentially inhabit the outer parts of the incoming galaxy, and thus be stripped off before the stars. Note that a fluctuation in the surface density of the GCs themselves would be challenging to detect at the $\sim 50 \%$ level.

As mentioned previously, there are no major signatures of interactions previously known for M87. Some candidates for large-scale, low-surface brightness stellar features have been identified (Arp \& Bertola 1971; Weil et al. 1997; Rudick et al. 2010), but these are at spatially disparate locations from the GC shell, and in some cases are likely to be manifestations of foreground cirrus.

More intriguingly, as mentioned above, there are five lowluminosity ellipticals within a $30-80 \mathrm{kpc}$ projected radius of M87: NGC 4476, NGC 4478, NGC 4486A, NGC 4486B, and IC 3443 (see Figures 1 and 12). Some of these have previously been proposed as the stripped remnants of larger galaxies, based on disturbances in their isophotes or unusual stellar colors for their luminosities. If any of these galaxies is being actively disrupted, some stars and GCs could have already been stripped out and deposited within the halo of M87.

The first four of these galaxies are in the ACSVCS sample, so we consider this scenario in more concrete terms by marking them in Figure 11 with red $\times$ symbols. Here we see from the top two panels that all four galaxies appear depleted in GCs relative to the average trend, which suggests stripping of their outer regions. The offsets are strongest for NGC 4486A and NGC 4486B, which are statistically consistent with hosting zero GCs (see further discussion in Peng et al. 2008). NGC 4486B is also a very unusual outlier in the $\sigma_{\mathrm{c}}-L_{V}$ panel, and possibly in supermassive black hole correlations (Gültekin et al. 2009), implying that not only its GCs but also 90\% of its stars have been stripped away.

The $\sigma_{\mathrm{c}}$ values of NGC 4486A and NGC 4486B suggest that they originally hosted $\sim 70-200$ and $\sim 100-500$ GCs, respectively. Their phase-space positions in Figure 1(b), where their velocities are $\sim 750 \mathrm{~km} \mathrm{~s}^{-1}$ and $\sim 1550 \mathrm{~km} \mathrm{~s}^{-1}$, respectively, indicate that NGC 4486B could be associated with the shell. NGC 4478 (at $\sim 1400 \mathrm{~km} \mathrm{~s}^{-1}$ ) is also consistent with the shell in phase space; it does not appear to be as severely stripped, but still could have lost up to $\sim 250 \mathrm{GCs}$.

The colors of the NGC 4478 GCs also provide one of the closer matches to the shell GC colors (this test is not possible for NGC 4486A and NGC 4486B since they have virtually no GCs remaining). For both NGC 4478 and NGC 4486B, the dynamical friction timescales at their projected radii are $\sim 1-3$ Gyr or shorter, reinforcing the likelihood that these are objects in the final stages of orbital decay toward the center of M87. For both galaxies, there are also outer stellar velocity dispersion measurements available, making the extrapolation to $\sigma_{\mathrm{GC}}$ less uncertain. For NGC 4478 this is $\sim 50-100 \mathrm{~km} \mathrm{~s}^{-1}$ (Halliday et al. 2001), and for NGC 4486B, it is $\sim 100-120 \mathrm{~km} \mathrm{~s}^{-1}$ (Kormendy et al. 1997; Spolaor et al. 2010).

The present-day luminosity of NGC $4478, \log \left(L_{V} / L_{V, \odot}\right)=$ 9.8 , suggests that it may have experienced very little stellar stripping (Figure 11, lower left), which would explain the lack of surface brightness variations around M87. For NGC 4486B, the implied luminosity at infall of $\log \left(L_{V} / L_{V, \odot}\right) \sim 10.1$ would 


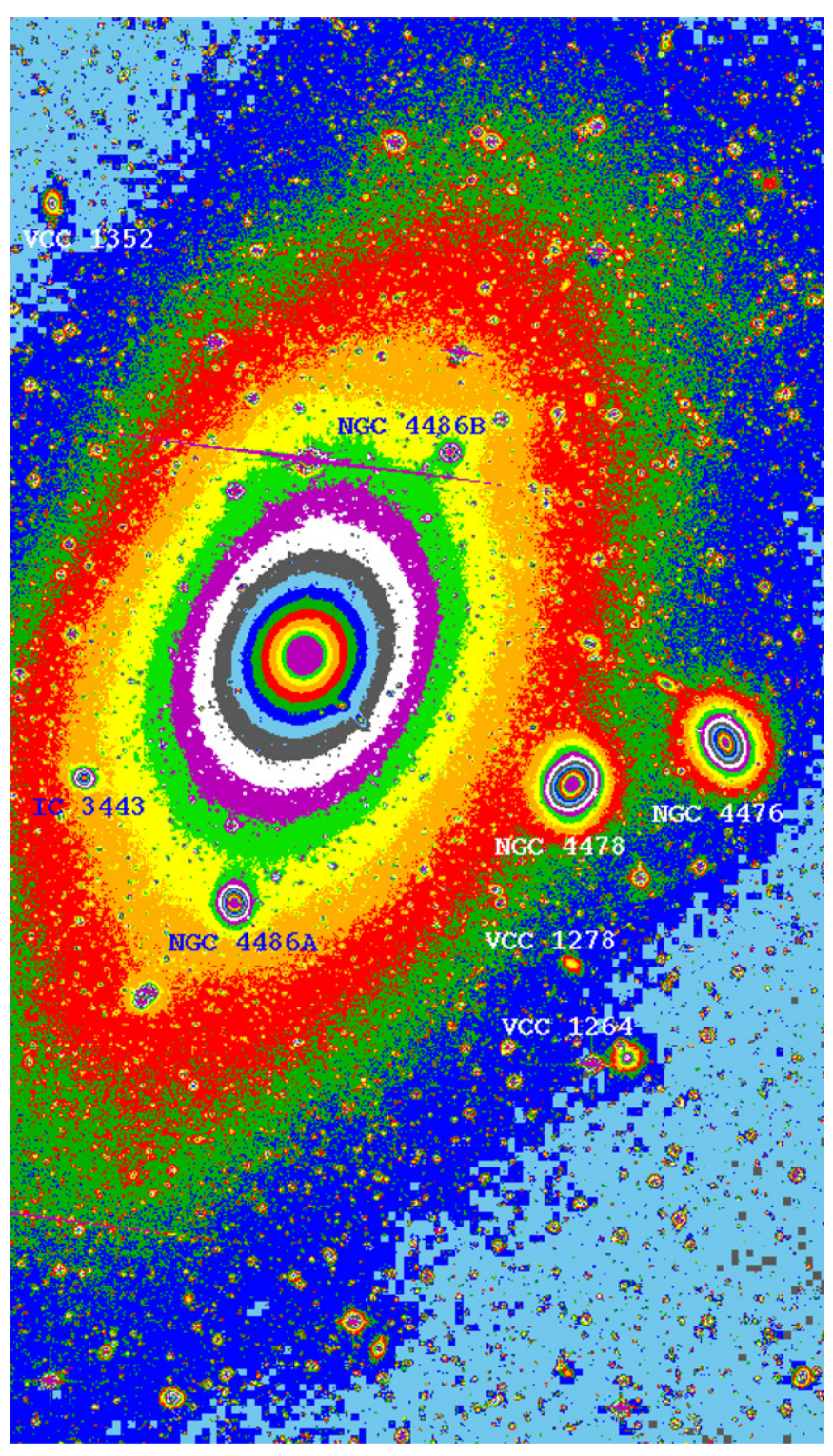

Figure 12. Closer view of the M87 $V$-band image from Figure 1(a), showing a $23^{\prime} .5 \times 40^{\prime}(110 \times 190 \mathrm{kpc})$ region. Some small galaxies in the field are labeled. The shading is logarithmically spaced by surface brightness and arbitrarily colored in order to enhance any isophote twisting (e.g., in the case of NGC 4478). The most probable shell GCs have positions spread along the outermost, dark-green and dark-blue isophotes, and preferentially in the northern half of the galaxy.

(A color version of this figure is available in the online journal.)

require that much of the stellar debris be hidden at small galactocentric radii, which is plausible given that this stripped galaxy is currently at a radius of $\sim 30 \mathrm{kpc}$ (note that the missing debris is an issue even without considering the GC shell).

Both of these galaxies appear to be possible shell progenitors, as their properties are consistent with all of the constraints discussed above, within the uncertainties, except for the lowvelocity dispersion in the chevron model (this dispersion could then be considered either as an artifact of the adopted model, or as a product of stripping dynamics that are not yet understood). In fact, the problem could be turned around: given that there are stripped galaxies around M87 which must have lost hundreds of accompanying GCs, should we not expect to see some evidence for them in phase space?
The multiplicity of candidate shell progenitors is also a reminder that our initial single-progenitor assumption may be oversimplified. It is indeed possible that the phase space of GCs in the M87 halo contains multiple, overlapping, prominent shells and streams. ${ }^{16}$ More GC spectra are clearly needed to better determine the substructure properties, and to make clearer progenitor inferences.

As a final plausibility check, we examine whether or not our preferred solution, with an accreted luminosity of $\log \left(L_{V} / L_{V, \odot}\right) \sim 10$, (i.e., $\sim 0.5 L^{*}$, or a $\sim 1: 10$ merger), represents a likely event in the context of other observational constraints on merger rates in BCGs. As discussed in Section 1, the details of BCG assembly are not well known, which motivates our phase-space study, but there are still some plausible upper limits on merger rates. For example, M87 cannot have accreted a galaxy with $\log \left(L_{V} / L_{V, \odot}\right) \sim 10$ more often than once every $\sim 1.5 \mathrm{Gyr}$, averaged over a Hubble time, or else the total luminosity would be higher than is observed today.

The calculation is more complicated than this because the merger history will be divided over a spectrum of mass ratios, and because the merger rate may change with time. We therefore turn to the low- $z$ BCG survey carried out by Liu et al. (2009), who found that major mergers (down to a $\sim 1: 4$ mass ratio) were visible in 1 case out of every 30 . If we assume for simplicity that mergers are detectable in phase space for roughly twice as long as in real space (which is the only place that merger timescales will enter into our calculations), then recent major merger signatures could be found in $\sim 7 \%$ of BCGs.

Next, we should like to extrapolate to lower-mass ratios. The simplest assumption we can make is that the accreted objects follow a standard Schechter luminosity function, where 1:4 events correspond to $\sim L^{*}$ in the case of M87. We then calculate that events down to 1:10 should be present in $\sim 25 \%$ of BCGs. Thus, it does not seem improbable that the shell in M87 traces a $\sim 1: 10$ merger event, while more massive progenitors would begin to stretch the limits of plausibility.

\section{OUTER STREAM ANALYSIS}

We next analyze the GCs in the northwest area, around "stream A." Figure 13 summarizes the positional-space and phase-space properties of this area. The known stellar stream has a $V$-band luminosity of $(2.4 \pm 0.3) \times 10^{8} L_{V, \odot}$ over a $\sim 15 \times 100 \mathrm{kpc}$ region (Janowiecki et al. 2010).

It is important to keep in mind that the spectroscopic observations were tiled closely around the stellar stream, and much of the apparent narrowness in real space of the overall GC distribution is an artifact of this bias. The fractional return of bona fide GCs from the spectroscopic observations was indeed higher "on-stream" than "off-stream," but the statistics here are relatively poor and a more in-depth analysis will be required to consider this question further.

We focus instead on a possible cold phase-space substructure within the spectroscopic sample, where the hotter background may be the stream or the general diffuse outer GC system of M87. This tentative substructure consists of six GCs (out of 19 in the sample apart from one associated with a nearby galaxy) that are clustered in real space and follow a cold diagonal path in phase space (drawn schematically in Figure 13(b)). Two of these objects are a very close pair, separated spatially by only

16 There may be another, related feature in M87: $\mathrm{S}+11$ identified by a peculiar velocity offset in the metal-rich GCs inside $\sim 10 \mathrm{kpc}$. 

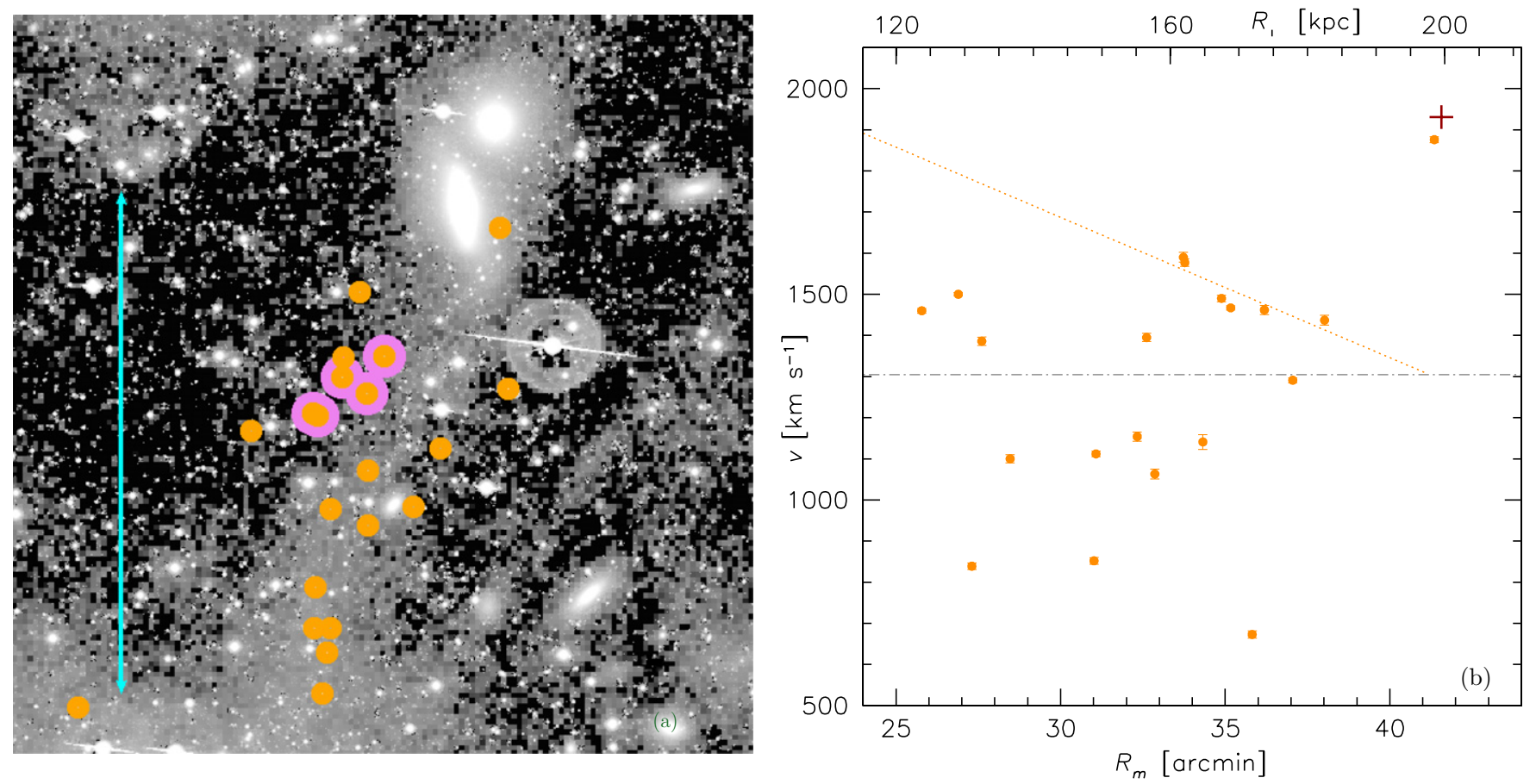

Figure 13. Globular clusters near outer "stream A." (a) Positional space (a zoom-in of Figure 1(a)). The five candidate stream GCs are outlined in purple (the two easternmost ones are almost overlapping). The bar with arrows again shows a $100 \mathrm{kpc}$ scale. (b) Phase space (using distance from the center of M87). Velocity uncertainties are indicated by error bars. One GC at upper right is probably bound to the low-luminosity S0 NGC 4461 (red cross). The systemic velocity is shown by a horizontal line and a diagonal line illustrates our best-fit stream model.

(A color version of this figure is available in the online journal.)

$2.6 \operatorname{arcsec}(0.2 \mathrm{kpc})$ and in velocity by only $13 \mathrm{~km} \mathrm{~s}^{-1}$, which is consistent with zero within the uncertainties.

To estimate the chances of this substructure being a chance clumping, we pursue friends-of-friends group finding as previously carried out for the shell (Section 3.2), but reverting to a traditional three-dimensional $(x, y, v)$ phase-space search. In order to pick up the apparent group in the real data, we adopt $w_{x}=w_{y}=50 \operatorname{arcsec}(4.0 \mathrm{kpc}), w_{v}=30 \mathrm{~km} \mathrm{~s}^{-1}$, and $\lambda=0.29$. We then simulate mock data sets using the same positions while drawing velocities from a Gaussian LOSVD with a dispersion of $271 \mathrm{~km} \mathrm{~s}^{-1}$ and find groups with six or more members by chance only $9 \%$ of the time. Again, this analysis is conservative in that the mock data sets start from spatial positions that may not be random, but is not conservative in that the group-finding parameters are optimized a posteriori.

We next carry out maximum-likelihood model fits to the data, using a Gaussian plus one-sided chevron model. We estimate that $4_{-2}^{+3}$ of the GCs in this region belong to the stream, which has a dispersion of $33_{-25}^{+11} \mathrm{~km} \mathrm{~s}^{-1}$. This model is intended as a characterization of the stream properties rather than as a robust test of its reality, since the fits are somewhat unstable to the starting conditions. The model does not incorporate the full spatial information about the region, and given that the stream objects are also clumped in the azimuthal direction, we consider it likely that the five most closely associated objects seen in Figure 13 comprise the stream. Extrapolating to fainter magnitudes from our spectroscopic limit of $i_{0} \sim 22.5$, we estimate a total of $\sim 15 \mathrm{GCs}$ in the stream clump. However, our spectroscopic survey is incomplete, and there might easily be $\sim 20-30$ stream GCs.

As with the shell analysis, we now consider the independent dimension of metallicity (using color as a proxy). Figure 14 shows that the stream GCs may have a different color

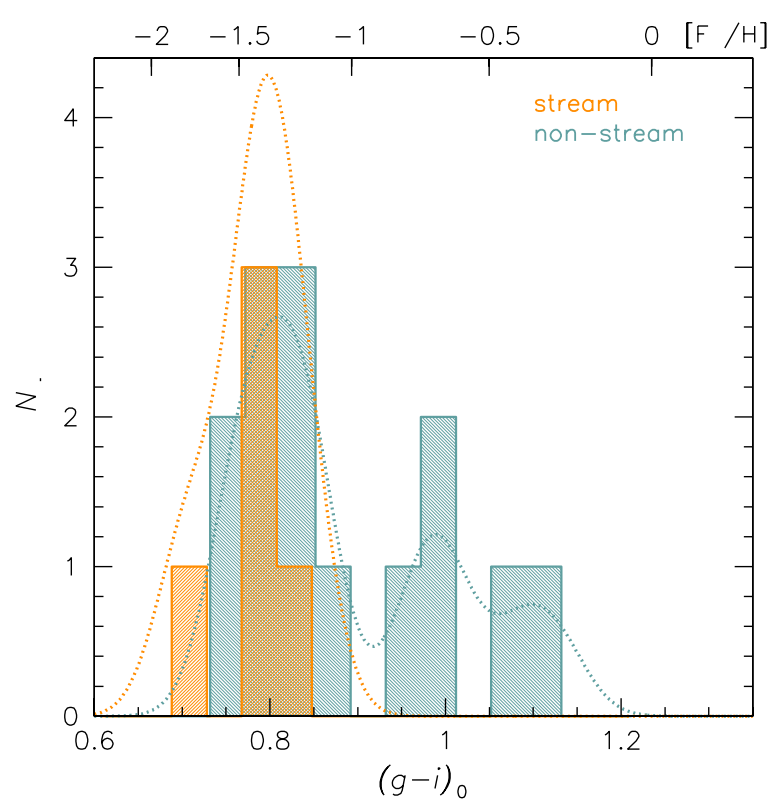

Figure 14. Color distribution of GCs in the stream A region. The two colored histograms show the stream and non-stream objects, as per the legend, with alternative smoothed distributions also shown (see Figure 10(b)). The stream GCs do not include any red objects, but the difference with the non-stream GCs is not statistically significant.

(A color version of this figure is available in the online journal.)

distribution from the rest of the GCs. The former are narrowly confined to a blue color range of $(g-i)_{0}=0.71-0.83$ (a range in $[\mathrm{Fe} / \mathrm{H}]$ of $\sim 0.5 \mathrm{dex})$, while the latter include a similar blue peak but also a second, much redder peak, with $(g-i)_{0}=0.97-1.13$. From the K-S test, this difference has a $22 \%$ chance of occurring 
Table 3

Parameters of Accretion Simulations

\begin{tabular}{lccc}
\hline \hline & Cold Shell & Cold Stream & Hot Shell \\
\hline Satellite effective radius, $R_{\mathrm{e}}(\mathrm{kpc})$ & 1 & 1 & 4 \\
Satellite velocity dispersion, $\sigma_{\mathrm{p}}\left(R_{\mathrm{e}}\right)\left(\mathrm{km} \mathrm{s}^{-1}\right)$ & 35 & 35 & 200 \\
Satellite mass, $M_{\mathrm{sat}}\left(M_{\odot}\right)$ & $1.8 \times 10^{9}$ & $1.8 \times 10^{9}$ & $3.2 \times 10^{11}$ \\
Particle number, $N_{\mathrm{p}}$ & 65,536 & 65,536 & 65,536 \\
Particle mass, $M_{\mathrm{p}}\left(M_{\odot}\right)$ & $2.75 \times 10^{4}$ & $2.75 \times 10^{4}$ & $4.9 \times 10^{6}$ \\
Gravitational softening length, $r_{\mathrm{l}}(\mathrm{pc})$ & 20 & 20 & 22 \\
Initial transverse velocity, $v_{\mathrm{t}} / v_{\mathrm{c}}$ & 0.05 & 0.1 & 0.1 \\
Initial apocentric radius, $r_{\mathrm{apo}}(\mathrm{kpc})$ & 90 & 200 & 90 \\
Initial pericentric radius, $r_{\mathrm{peri}}(\mathrm{kpc})$ & 2 & 12 & 4.7 \\
Host dark halo virial mass, $M_{200}\left(M_{\odot}\right)$ & $4.2 \times 10^{14}$ & $4.2 \times 10^{14}$ & $4.2 \times 10^{14}$ \\
Host dark halo virial radius, $r_{200}(\mathrm{Mpc})$ & 1.55 & 1.55 & 1.55 \\
Host dark halo scale radius, $r_{\mathrm{s}}(\mathrm{Mpc})$ & 0.564 & 0.564 & 0.564 \\
Host stellar effective radius, $R_{\mathrm{e}}(\mathrm{kpc})$ & 7 & 7 & 7 \\
Host circular velocity at $R_{\mathrm{e}}, v_{c}(\mathrm{~km} \mathrm{~s})$ & 475 & 475 & 475 \\
Time step, $t_{\mathrm{s}}(\mathrm{Myr})$ & 0.23 & 0.23 & 0.14 \\
\hline
\end{tabular}

randomly. As with the shell, the color information of the stream provides independent confirmation of its distinctiveness.

As discussed for the shell region, we could in principle connect the peak color of the stream GCs to a possible progenitor, but the statistics are currently too poor for strong constraints. Even so, we can still mention one example of a potential analogue to the stream progenitor: the Virgo dwarf elliptical IC 3328, with a stellar dispersion of $\sim 35 \mathrm{~km} \mathrm{~s}^{-1}$, hosting $\sim 40$ GCs with a color peak near $(g-z)_{0} \sim 0.92$, i.e., $(g-i)_{0} \sim 0.77$.

Could this apparent GC stream from a dwarf galaxy be associated with the previously identified stellar stream? The latter has a luminosity of $\log \left(L_{V} / L_{V, \odot}\right) \sim 8.4$ that is consistent with this hypothesis (compare the $L_{V}-\sigma_{\mathrm{c}}$ trends for ACSVCS galaxies in the lower left panel of Figure 11). Its color of $(B-V) \sim 0.8$ also suggests a dwarf progenitor (Rudick et al. 2010), although more massive progenitors are also possible within the uncertainties.

A puzzle is presented by the spatial offset of $\sim 10 \mathrm{kpc}$ between the stellar and GC substructures (Figure 13(b)). One possibility that will require further modeling is that the GCs and stars followed different trajectories owing to their different initial binding energies. The nucleus of the dwarf could also very well still be visible around M87, although we have not identified an obvious candidate.

Another interesting possibility is that both the stream and the shell are part of the same accretion event, since their peak GC colors and velocity dispersions are similar. Exploring this scenario will require more extensive simulations than we can attempt here.

\section{THEORETICAL ANALYSIS}

We now attempt to model the dynamics of the observed substructures, based mostly on numerical simulations but also making use of analytic representations of orbit kinematics.

The narrow diagonal tracks of the stream and shell in phase space can be understood in simple terms as the near-radial infall of objects with similar initial potential energies. Analogous features are found in Local Group accretion events (Gilbert et al. 2007) and in simulations of minor mergers (e.g., Quinn 1984; Dupraz \& Combes 1986; Fardal et al. 2007).

The phase-space structure of tidally stripped material can be influenced by many factors (e.g., Hernquist \& Quinn 1988, 1989; Johnston 1998; Helmi et al. 1999; Johnston et al. 2002;
Helmi 2004; Rudick et al. 2009), which we summarize in broad terms as follows. More massive satellites produce more diffuse streams owing to the higher internal velocity dispersions and larger sizes of these systems. In cuspy potentials, low angular momentum orbits can bring the satellites close to the potential center and lead to broader shells or fan-like features, while in orbits farther out, the weaker tidal shear produces narrower loops and streams. Finally, any dynamically cold substructure will tend to diffuse over time due either to nonaxisymmetry in the host potential or to perturbations from other satellites.

As a proof of principle, we carry out a basic set of simulations inspired by M87 and its substructures. These are not intended to explore the (very large) orbital and progenitor parameter space for the infalling satellite galaxy, or to provide a unique, robust match to the data, but to illustrate qualitatively how comparable features can emerge in phase space from the tidal stripping of infalling galaxies.

We conduct $N$-body simulations of self-gravitating low-mass galaxies orbiting in a fixed spherical gravitational potential. For this cluster-mass potential, we use a Navarro-Frenk-White model for the dark matter halo (Navarro et al. 1996; McLaughlin 1999), and embed a Hernquist (1990) profile to represent the stellar mass of the central galaxy. The relevant parameters are reported in Table 3.

For the inner shell, the main features that we wish to reproduce are the apparent chevron morphology in phase space including a low velocity dispersion, with a high degree of azimuthal mixing in real space (see Figure 1). For the outer stream, the goal is to find a thin structure in both real and phase space, which is not closely connected to the progenitor galaxy or nucleus.

For both features, we adopt the same low-mass galaxy model for the infalling satellite, and consider a modest number of orbital configurations. The infalling satellite galaxy follows a single-component Hernquist model, with characteristic parameters summarized in Table 3. A real galaxy will consist of multiple components (stars, GCs, gas, and dark matter), but this introduces additional degrees of freedom to the problem which we are for now keeping as conceptually simple as possible.

We place the satellite on elongated orbits around the potential center, ${ }^{17}$ and run the simulations using an $\mathrm{N}$-body treecode

\footnotetext{
17 Our adopted apocentric distances of $\sim 100-200 \mathrm{kpc}$ may not seem consistent with satellites that fall in from the large-scale environment, but our goal is to set up an idealized model of accretion dynamics once an object has migrated inward by scattering or dynamical friction (e.g., Faltenbacher \& Mathews 2005)
} 
(Hernquist 1987), which simulates the fully self-gravitating response of the infalling satellite. The simulations were evolved for several Gyr using a fixed time step of $0.25 \mathrm{Myr}$. We create real-space and phase-space plots of all the satellite particles for each time step, using a small selection of viewing angles. We then select by eye the best combination of time step and viewing angles that qualitatively resembles the data.

Next, we sample a subset of the particles to represent the GC observations, plotting $10^{4}$ particles to obtain a general sense of the substructure morphology, and an additional subsample of 100 particles for more direct comparison with the data. Our default selection consists of an unbiased, random sample, which can be considered as representing the limiting case where most of the dark halo has been stripped away, with the remaining selfgravitating material traced well by the stars and GCs. In almost every real galaxy, the metal-poor GCs occupy a more extended distribution than the field stars, so we have tried the alternative limiting case where the least bound particles are selected as "GCs." The results from the two sampling schemes turn out to be qualitatively similar.

Clearly, our procedures are only the initial steps toward modeling the M87 halo kinematics. Ideally, one would like to: use a live, triaxial potential that includes the effects of dynamical friction and other substructures; explore a wide range of satellite and orbital parameters; and use an objective, quantitative metric for optimizing the model fits. However, our current approach is adequate for providing a broad sense of some of the types of substructure signatures that are physically plausible, which one can see in related analyses of other systems (Hernquist \& Quinn 1988; Merrifield \& Kuijken 1998; Harding et al. 2001; Bullock \& Johnston 2005; Gilbert et al. 2007; Fardal et al. 2007; Peñarrubia et al. 2010).

The best qualitative matches that we identified for the shell and the stream (separately) were shown in Figure 2. The shell progenitor made a close passage to the center of M87 and was largely disrupted, leaving a classic chevron signature in phase space, while also being spread out in positional space. The stream progenitor has a larger pericenter and is disrupted more gradually, maintaining integrity as a narrow stream in both phase space and positional space for many orbits.

In a real system, any triaxiality of the potential would cause the orbits to phase mix more rapidly, and the time evolution of the mass distribution would presumably smear out the cold substructures even further (as well as inhibit any orbital resonances that might in principle be an alternative mechanism for producing cold kinematical features). Simulations of clusterscale tidal streams in a cosmological context (Rudick et al. 2009) indicate that streams are disrupted over timescales of $\sim 2-4$ times the local dynamical timescale, $t_{\mathrm{dyn}} \sim r / v_{\mathrm{c}}$. For the shell and stream, $t_{\mathrm{dyn}} \sim 0.2$ and $0.5 \mathrm{Gyr}$, respectively, so their most likely lifetimes are $\sim 0.5 \mathrm{Gyr}$ and $\sim 1.5 \mathrm{Gyr}$.

One important feature of coherent substructures is that they trace out a close family of orbits over large scales in galaxy halos, and can thus be used to provide unique constraints on the gravitational potential, including the radial distribution and shape of the dark matter halo (e.g., Law et al. 2009; Koposov et al. 2010; Varghese et al. 2011). The case of a spherical system is conceptually simple: the potential energy lost by particles as they fall in toward the center of the host galaxy is reflected in a monotonic trend of increasing velocities with decreasing radius, modulo projection effects (Merrifield \& Kuijken 1998).

We derive a new formulation of this method, assuming a spherical gravitational field of a power-law form, where the

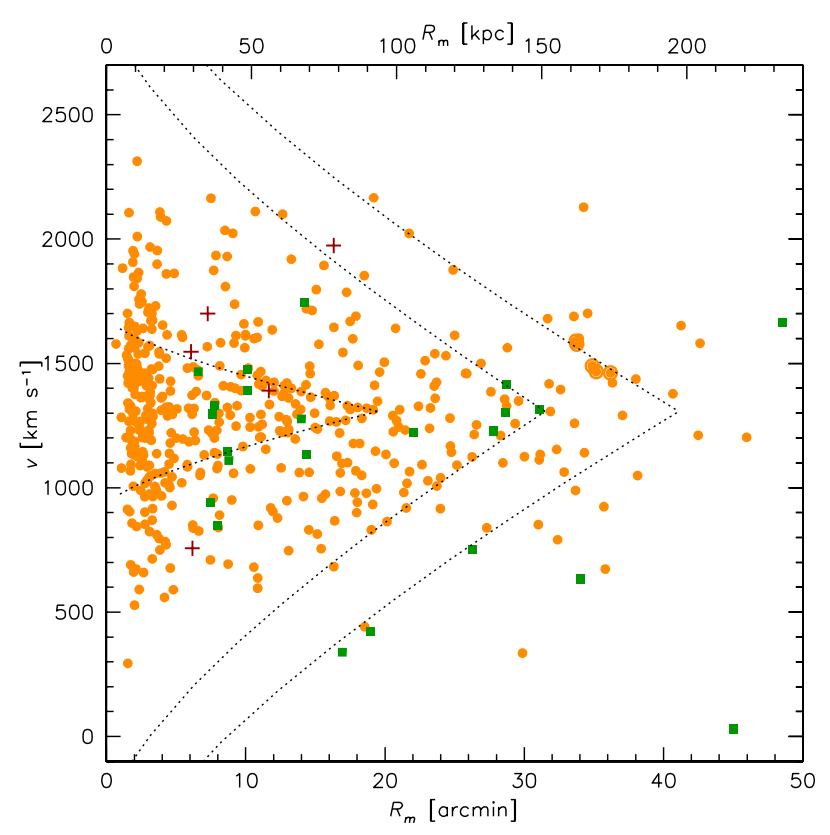

Figure 15. Phase-space diagram of M87 as in Figure 1(b), with three monoenergetic model curves overplotted (see the text for details). GC and satellite galaxy data are shown by orange dots and red crosses as before, with planetary nebulae (Doherty et al. 2009) also now included as green squares. The outer stream subgroup of GCs (at $R_{m} \sim 35 \mathrm{arcmin}$ ) is highlighted with larger circles. The inner shell and outer stream GCs can be represented well by model curves as shown, and there is a hint of an intermediate-radius shell as marked, whose apex could explain the low velocity dispersion found in a subgroup of the PNe.

(A color version of this figure is available in the online journal.)

total density, circular velocity, and potential are

$$
\begin{gathered}
\rho_{\mathrm{tot}} \propto r^{-\alpha}, \\
v_{\mathrm{c}}=v_{0}{\frac{r}{r_{0}}}^{1-\frac{\alpha}{2}}, \\
\Phi(r)=\frac{v_{0}^{2}}{2-\alpha}{\frac{r}{r_{0}}}^{2-\frac{\alpha}{2}},
\end{gathered}
$$

where $\alpha<2$, as is probably appropriate on $\sim 10-100 \mathrm{kpc}$ scales in M87 (see also Section 2.2(f) of Binney \& Tremaine 2008). Following Equation (3) of Merrifield \& Kuijken (1998), we consider a thin shell of radius $r_{\text {apo }}$ where particles have zero velocity (i.e., they are on radial orbits at apocenter). By conservation of energy and by geometry, their line-of-sight velocities at three-dimensional radius $r$ and two-dimensional radius $R$ are then given by

$$
v_{\mathrm{LOS}}^{2}=2 \quad 1-\frac{R^{2}}{r^{2}} \quad\left[\Phi\left(r_{\mathrm{apo}}\right)-\Phi(r)\right] .
$$

The velocity "edge" of the shell is then determined by solving for the maximum of $v_{\mathrm{LOS}}$ at a given radius $R$. By fitting this simple model to a cold phase-space feature, we can then estimate

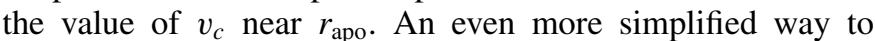
understand this constraint (Merrifield \& Kuijken 1998) is that the slope of the shell velocity edge with radius in phase space is approximately equal to $v_{\mathrm{c}} / r_{\text {apo }}$. In practice, the dominant effect is the change of potential energy, so the determination of $v_{c}$ is fairly insensitive to the form adopted for $\Phi(r)$.

In Figure 15, we show this model applied to the stream and to two possible shells in M87, where we have taken the maximumlikelihood linear-slope fits from Sections 3.4 and 4 as starting 

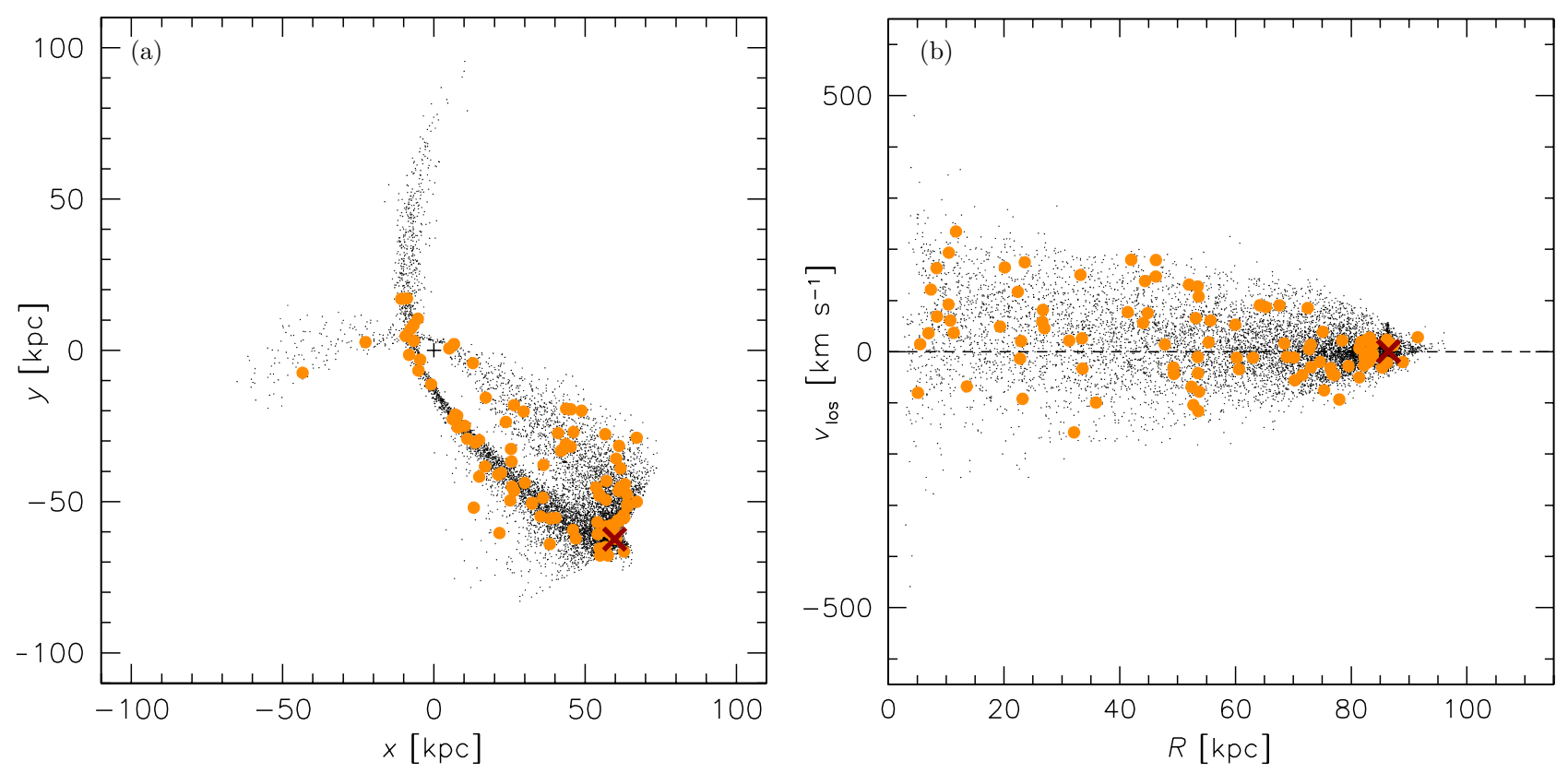

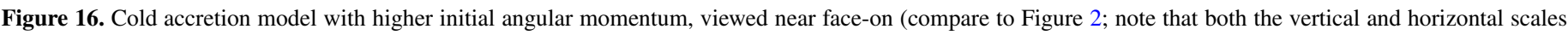

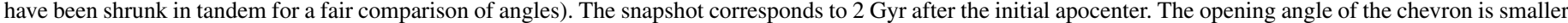
than in Figure 2(b), and in position space the stream stays highly coherent for many orbits (in the absence of perturbations in the potential).

(A color version of this figure is available in the online journal.)

points for approximate fits in phase space. The model reproduces nicely the observed radial trends and suggests some possible connections in phase space between the shells and nearby lowluminosity ellipticals. Also shown in the figure are halo $\mathrm{PNe}$, which show hints of tracing some of the same substructures seen in the GCs. More precise orbital fits to the substructures and the potential progenitors will require dedicated dynamical modeling (e.g., Romanowsky \& Kochanek 2001; Murphy et al. 2011).

There is a hitch with these model curves: the implied circular velocities differ radically from typical estimates for the M87 potential. At $r \sim 20,32,41 \operatorname{arcmin}(\sim 90,150$, $200 \mathrm{kpc}$ ), we infer $v_{\mathrm{c}} \sim 270,1200,1400 \mathrm{~km} \mathrm{~s}^{-1}$, albeit with substantial uncertainties. Previous estimates from dynamical and X-ray analyses yielded $v_{\mathrm{c}} \sim 650-900 \mathrm{~km} \mathrm{~s}^{-1}$ over this entire radial range (Das et al. 2010; Murphy et al. 2011), while our own equilibrium-based dynamical analysis indicates $v_{\mathrm{c}} \sim 400-550 \mathrm{~km} \mathrm{~s}^{-1}(\mathrm{~S}+11) .{ }^{18}$ These discrepancies should not be a major concern at this point because there are various complications to consider including the radius convention of Section 3.1, as well as non-spherical effects (Jílková et al. 2010). In particular, a planar orbit viewed near face-on will show depressed line-of-sight velocities, and a reduced opening angle of the chevron.

An illustration of the latter effect is provided in Figure 16, which shows an alternative orbit for the shell that has higher angular momentum than our fiducial case and maintains better planar integrity. Here the "measured" $v_{c}$ would be $\sim 250 \mathrm{~km} \mathrm{~s}^{-1}$, despite the true value in the model being $635 \mathrm{~km} \mathrm{~s}^{-1}$. Note that the simulation shown is also representative of a large family of

\footnotetext{
18 The local escape velocity is at least $v_{\mathrm{e}}=2^{1 / 2} v_{\mathrm{c}}$. We regard the $v_{\mathrm{c}} \sim 550 \mathrm{~km} \mathrm{~s}^{-1}$ model as plausibly describing a massive group-sized dark matter halo around M87, in which case $v_{\mathrm{e}} \gtrsim 800 \mathrm{~km} \mathrm{~s}^{-1}$. This would indicate a bound-velocity range of $\sim 500-2100 \mathrm{~km} \mathrm{~s}^{-1}$, and probably wider, suggesting that most or all of the objects in Figure 15 are bound to M87 (modulo the unknown proper-motion velocities).
}

orbits that are not a good match to the shell observations because of the high degree of spatial coherence.

As discussed at length in Section 3.6, there is an apparent conflict between the shell's inferred kinematical coldness and the large number of GCs, since these imply very different progenitor masses. We consider this issue more explicitly by changing the simulated shell progenitor to correspond to a more massive galaxy, with parameters summarized in Table 3.

We show an example post-disruption snapshot in Figure 17, finding as expected that the resulting shell kinematics are hotter than in the dwarf-galaxy simulation, and do not compare as favorably to the observations (compare Figures 2(b) and 3(b)). However, as previously discussed, this tension is alleviated if we modify our default radius convention and chevron-based model (compare Figure 3(a)).

These simulations bracket the simple cases of very low- and high-mass progenitor galaxies, but a worthwhile endeavor for the future would be simulating more detailed intermediate-mass cases, including bulge, disk, stellar halo, and dark matter halo subcomponents. An additional aspect that could be pursued is the modeling of velocity gradients with azimuthal angle, which should be generically found in tidal features (e.g., Helmi et al. 2003). Our initial look at the M87 shell data shows no clear sign of such effects.

\section{SUMMARY}

We have used an unprecedentedly wide-field data set of high-precision GC velocities to search for phase-space substructure around the central cluster galaxy M87. The large majority of the data are from $\mathrm{S}+11$, with a few additional velocities obtained from the literature, and from a new sample of Keck/DEIMOS spectroscopy presented here that targets a known filament of stellar light in the outer halo. Analysis of $(g-i)$ colors and spectroscopic metallicities for a subset of our GC sample confirms that the colors provide a good proxy for metallicity, allowing for chemical tagging of substructures. 

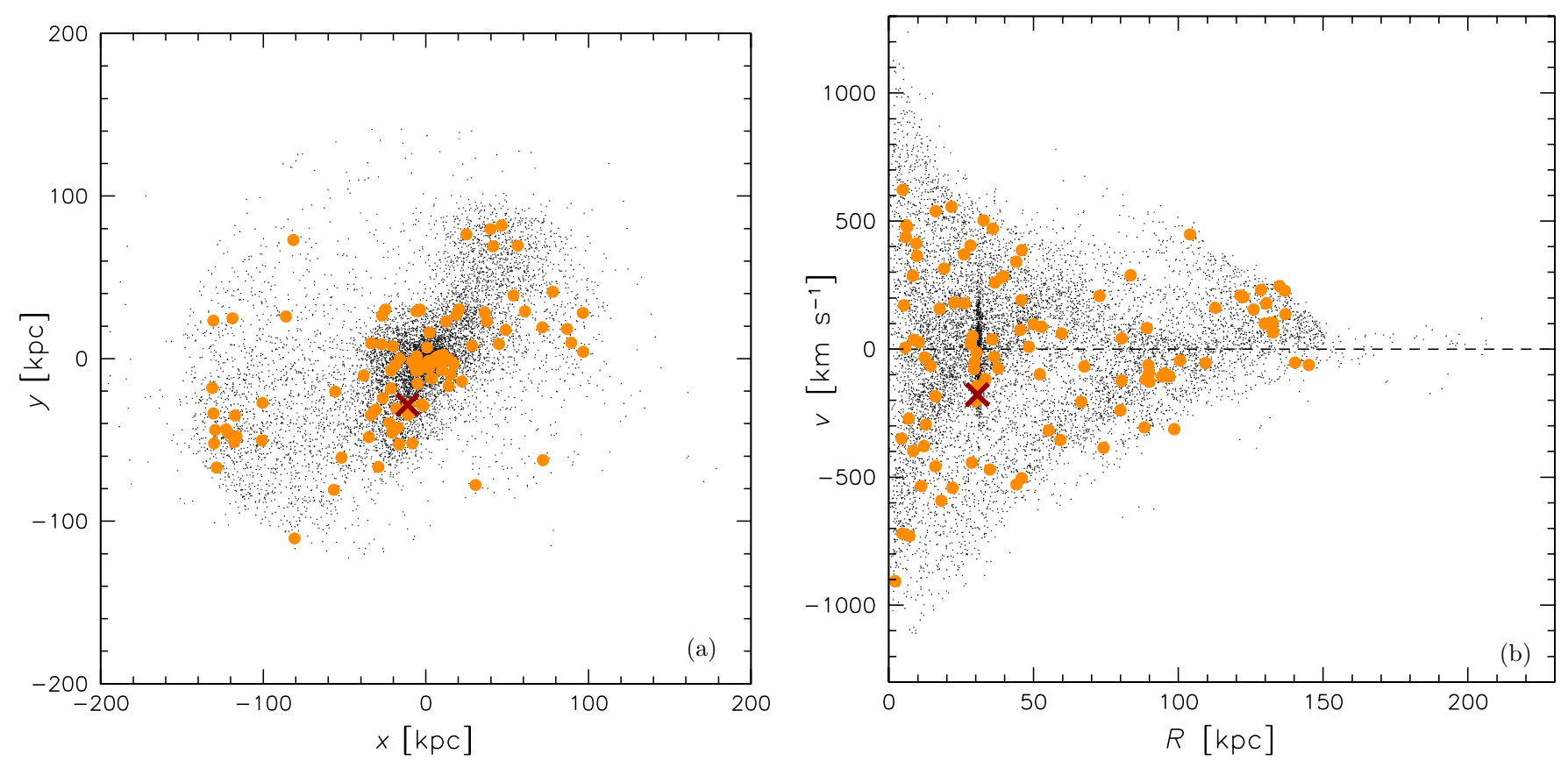

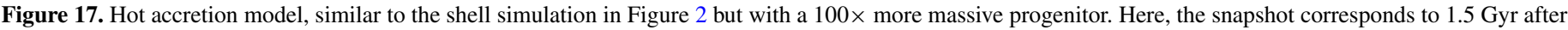
the initial apocenter. Similar features are present as with the low-mass galaxy but are less distinct.

(A color version of this figure is available in the online journal.)

We identify two candidate features: one a small stream near to the outer-halo stellar filament, and the other a previously unknown colossal "shell" in the inner halo. We have carried out extensive statistical tests and characterizations of these substructures.

The outer GC stream appears to be a collection of $15 \pm 10$ objects (after extrapolating to fainter magnitudes) residing in a $\sim 5 \times 20 \mathrm{kpc}$ clump with an internal velocity dispersion of $\sim 30 \pm 20 \mathrm{~km} \mathrm{~s}^{-1}$. We have found the presence of this stream to be significant both in a group-finding analysis $(p=0.09)$, from a maximum-likelihood model $(p=0.025)$, and from its distinctive color distribution compared to the surrounding GCs $(p=0.22)$.

The stream dispersion, its GC colors and numbers counts, the color of the stellar filament, and the filament's narrowness all suggest the accretion of a dwarf galaxy. Low-mass kinematical substructures in elliptical galaxies have been inferred in other studies (Côté et al. 2003; Woodley \& Harris 2011), but in this case the association with a visible counterpart should allow for a much clearer determination of the accretion dynamics.

The shell of GCs has a chevron-like shape in phase space that resembles classic expectations for a disrupted infalling system (Hernquist \& Quinn 1988), although the interpretation in detail is more challenging. The appearance of the shell is sharpened by a change of coordinates from simple radius to elliptical, circularequivalent radius. The significance of the shell in phase space is established using a group-finding algorithm ( $p<0.01)$, a crude entropy metric $(p=0.31)$, and a maximum-likelihood chevron-based model ( $p<0.01$ compared to a pure Gaussian model). The color distribution of the shell and non-shell GCs from the same region is different $(p=0.13)$, providing independent evidence for the existence of the shell. The phasespace morphology resembles a simple chevron model more than a wedge or tapered Gaussian, but may very well have a complex, intermediate morphology; however, such uncertainties do not compromise our basic finding of a relatively massive and cold substructure.

We find from maximum-likelihood fitting that the shell contains $500-1100$ GCs, which is $\sim 5$ times the entire Milky Way system, and comprises around 20\% of M87's GCs in the 50-95 kpc region. This number suggests a stellar debris field of $(2-6) \times 10^{10} L_{\odot}$, brought in by either a large group of dwarf galaxies, or by a single giant elliptical or lenticular galaxy.

The dramatic appearance of the shell in phase space is due to its relatively cold kinematics, with an estimated velocity dispersion of $10-30 \mathrm{~km} \mathrm{~s}^{-1}$ (or up to $\sim 120 \mathrm{~km} \mathrm{~s}^{-1}$ if we make generous allowances for uncertainties concerning the shell morphology). The lower velocity dispersion estimates are difficult to reconcile with the number of inferred GCs, as they most naturally imply a dwarf galaxy hosting only a handful of GCs. However, after allowing for systematic uncertainties in our analyses, we suggest a solution involving an $\mathrm{E} / \mathrm{S} 0$ progenitor with a luminosity of $\sim 0.5 L^{*}$, which could very well also be the parent galaxy for one of the tidally stripped galaxies now visible close in to M87.

There is a critical need for additional data from the shell region, including new velocities of both GCs and $\mathrm{PNe}$, in order to confirm the presence of the substructure and to refine our estimates of its characteristics. Along these lines, we have recently obtained another cycle of GC spectroscopy from MMT/Hectospec that includes $\sim 35$ velocity measurements of independent objects in the shell region. A preliminary analysis yields no obvious sign of substructure, but the statistics from the new data are poor, and at least another $\sim 100$ velocities are needed.

We have carried out simplified $N$-body simulations of satellite galaxy accretion in a static cluster + BCG potential, finding that these readily produce streams and chevrons in phase space. In one simulation, a dwarf galaxy has recently fallen in and is partially disrupted, forming a classic, narrow tidal stream in both positional and phase space. In a second case, the initial 
infall orbit had much lower angular momentum, so that the satellite galaxy passed close to the center of M87 and was quickly disrupted. Its remnants are strewn more haphazardly across positional space and could be difficult to discern against the main body of M87, while a distinct chevron persists in phase space, with the apex corresponding to a shell turning point.

These simulations compare favorably to the M87 observations because the low internal velocity dispersions of the dwarf progenitors result in cold kinematics for the tidal debris. Simulations of a more massive progenitor produce hotter kinematics that do not as strongly resemble the data for the "shell," reiterating the tension between the different constraints on the shell progenitor mass, which remains unresolved pending further observations and modeling. It would also be worth investigating alternative explanations for the substructure, such as a shell-like boundary between well-mixed GC subpopulations formed or accreted in different epochs.

We have explored the use of the velocity-radius slopes of the substructures in phase space to estimate the underlying gravitational potential and dark matter distribution around M87. However, the interpretation of the data appears to be complicated by non-spherical effects.

Taken at face value, the shell GCs imply a substantial, recent accretion event in the halo of M87. This is the first large substructure identified around a central cluster galaxy, the first large stellar substructure with a clear kinematical detection in any type of galaxy beyond the Local Group, and by far the largest shell or stream discovered in the Local Universe.

M87 was a favored target for our survey as well as previous ones because of its proximity, rich GC system, and general lack of obvious dynamical disturbance. The large shell we have discovered is an example of the lurking accretion signatures that could be found from detailed phase-space studies of apparently placid galaxies.

There are however more indirect lines of evidence for a recent minor merger in M87, such as an off-center supermassive black hole (Batcheldor et al. 2010), a lopsided central GC velocity distribution $(\mathrm{S}+11)$, an accretion powered jet, and several surrounding peculiar low-luminosity satellites that could be the nuclei of since-shredded disk galaxies. Further theoretical work is needed to see if these features could all be explained by a unified scenario involving a recent gas-rich minor merger.

The shell and stream in M87 have probable lifetimes of $\sim 0.5$ Gyr and $\sim 1.5$ Gyr, respectively. Given our best guesses for the progenitors of these features, the total luminosity of M87 would be built up by $\sim 10$ or $\sim 1000$ events corresponding to the shell or the stream, respectively. If these recent events are representative of the long-term evolution of M87, and by extension of the class of BCGs in general, then they support a picture of late-epoch growth by hierarchical assembly (De Lucia \& Blaizot 2007).

More generally, the M87 results are another demonstration of the potency of GCs for providing unique information about the stellar halos of galaxies. We note the concluding prediction of Naab et al. (2009) for their landmark two-phase galaxy formation scenario: "... the outer parts of massive giant ellipticals will tend to be old, blue, metal-poor, and relatively uniform from galaxy to galaxy since they are all composed essentially of the debris from tidally destroyed accreted small systems." GCs have in fact long provided evidence for exactly this scenario.

We thank Nelson Caldwell for assistance with observations; Jason X. Prochaska and Kate Rubin for software help;
Magda Arnaboldi for providing results in electronic form; Lars Hernquist, Bill Mathews, and Mike Merrifield for helpful discussions; Vincenzo Pota for sharing his analyses in preparation; and Eric Emsellem for a constructive review.

J.B. and A.J.R. acknowledge support from the NSF through grants AST-0808099, AST-0909237, AST-1101733, and AST1109878, and by the UCSC University Affiliated Research Center's Aligned Research Program. J.S. was supported by NASA through a Hubble Fellowship, administered by the Space Telescope Science Institute, which is operated by the Association of Universities for Research in Astronomy, Incorporated, under NASA Contract NAS5-26555. J.C.M. has been supported by the NSF through grants AST-0607526 and AST-0707793. We acknowledge financial support from the Access to Major Research Facilities Programme, a component of the International Science Linkages Programme established under the Australian Government's innovation statement, Backing Australia's Ability.

Much of the data presented herein were obtained at the W. M. Keck Observatory, which is operated as a scientific partnership among the California Institute of Technology, the University of California, and the National Aeronautics and Space Administration. The Observatory was made possible by the generous financial support of the W. M. Keck Foundation. Observations reported here were obtained at the MMT Observatory, a joint facility of the Smithsonian Institution and the University of Arizona. Based in part on data collected at Subaru Telescope (which is operated by the National Astronomical Observatory of Japan), via a Gemini Observatory time exchange (GN-2009A-C-204). This research used the facilities of the Canadian Astronomy Data Centre operated by the National Research Council of Canada with the support of the Canadian Space Agency. We acknowledge the usage of the HyperLeda database (http://leda.univ-lyon1.fr). This research has made use of the NASA/IPAC Extragalactic Database (NED) which is operated by the Jet Propulsion Laboratory, California Institute of Technology, under contract with the National Aeronautics and Space Administration. Based on observations made with the NASA/ESA Hubble Space Telescope, and obtained from the Hubble Legacy Archive, which is a collaboration between the Space Telescope Science Institute (STScI/NASA), the Space Telescope European Coordinating Facility (ST-ECF/ESA), and the Canadian Astronomy Data Centre (CADC/NRC/CSA).

\section{REFERENCES}

Arnold, J. A., Romanowsky, A. J., Brodie, J. P., et al. 2011, ApJ, 736, L26 Arp, H., \& Bertola, F. 1971, ApJ, 163, 195

Ascaso, B., Aguerri, J. A. L., Varela, J., et al. 2011, ApJ, 726, 69

Ashman, K. M., Bird, C. M., \& Zepf, S. E. 1994, AJ, 108, 2348

Batcheldor, D., Robinson, A., Axon, D. J., Perlman, E. S., \& Merritt, D. 2010, ApJ, 717, L6

Bellazzini, M., Ferraro, F. R., \& Ibata, R. 2003, AJ, 125, 188

Belokurov, V., Zucker, D. B., Evans, N. W., et al. 2006, ApJ, 642, L137

Bergond, G., Zepf, S. E., Romanowsky, A. J., Sharples, R. M., \& Rhode, K. L. 2006, A\&A, 448, 155

Bernardi, M. 2009, MNRAS, 395, 1491

Binney, J., \& Tremaine, S. 2008, Galactic Dynamics (2nd ed.; Princeton, NJ: Princeton Univ. Press)

Brodie, J. P., Romanowsky, A. J., Strader, J., \& Forbes, D. A. 2011, AJ, 142, 199

Brodie, J. P., \& Strader, J. 2006, ARA\&A, 44, 193

Brough, S., Tran, K.-V., Sharp, R. G., von der Linden, A., \& Couch, W. J. 2011, MNRAS, 414, L80

Buitrago, F., Trujillo, I., Conselice, C. J., et al. 2008, ApJ, 687, L61

Bullock, J. S., \& Johnston, K. V. 2005, ApJ, 635, 931

Cassata, P., Giavalisco, M., Guo, Y., et al. 2011, ApJ, 743, 96 
Chilingarian, I. V. 2009, MNRAS, 394, 1229

Coccato, L., Gerhard, O., \& Arnaboldi, M. 2010, MNRAS, 407, L26

Coccato, L., Gerhard, O., Arnaboldi, M., et al. 2009, MNRAS, 394, 1249

Cohen, J. G., Blakeslee, J. P., \& Ryzhov, A. 1998, ApJ, 496, 808

Cohen, J. G., \& Ryzhov, A. 1997, ApJ, 486, 230

Collins, C. A., Stott, J. P., Hilton, M., et al. 2009a, Nature, 458, 603

Collins, M. L. M., Chapman, S. C., Irwin, M., et al. 2009b, MNRAS, 396, 1619

Cooper, A. P., Martínez-Delgado, D., Helly, J., et al. 2011, ApJ, 743, L21

Cortesi, A., Merrifield, M. R., Arnaboldi, M., et al. 2011, MNRAS, 414, 642

Côté, P., Marzke, R. O., \& West, M. J. 1998, ApJ, 501, 554

Côté, P., McLaughlin, D. E., Cohen, J. G., \& Blakeslee, J. P. 2003, ApJ, 591, 850

Damjanov, I., McCarthy, P. J., Abraham, R. G., et al. 2009, ApJ, 695, 101

Das, P., Gerhard, O., Churazov, E., \& Zhuravleva, I. 2010, MNRAS, 409, 1362

De Lucia, G., \& Blaizot, J. 2007, MNRAS, 375, 2

Doherty, M., Arnaboldi, M., Das, P., et al. 2009, A\&A, 502, 771

Dupraz, C., \& Combes, F. 1986, A\&A, 166, 53

Durrell, P. R., Mihos, J. C., Feldmeier, J. J., Jacoby, G. H., \& Ciardullo, R. 2003, ApJ, 582, 170

Evstigneeva, E. A., Gregg, M. D., Drinkwater, M. J., \& Hilker, M. 2007, AJ, 133,1722

Faltenbacher, A., \& Mathews, W. G. 2005, MNRAS, 362, 498

Fardal, M. A., Guhathakurta, P., Babul, A., \& McConnachie, A. W. 2007, MNRAS, 380, 15

Feldmeier, J. J., Ciardullo, R., Jacoby, G. H., \& Durrell, P. R. 2003, ApJS, 145 , 65

Forbes, D. A., \& Bridges, T. 2010, MNRAS, 404, 1203

Forbes, D. A., Spitler, L. R., Strader, J., et al. 2011, MNRAS, 413, 2943

Forte, J. C., Martinez, R. E., \& Muzzio, J. C. 1982, AJ, 87, 1465

Foster, C., Forbes, D. A., Proctor, R. N., et al. 2010, AJ, 139, 1566

Foster, C., Spitler, L. R., Romanowsky, A. J., et al. 2011, MNRAS, 415, 3393

Gao, S., Jiang, B.-W., \& Zhao, Y.-H. 2007, CJAA, 7, 111

Geisler, D., Wallerstein, G., Smith, V. V., \& Casetti-Dinescu, D. I. 2007, PASP, 119,939

Gilbert, K. M., Fardal, M., Kalirai, J. S., et al. 2007, ApJ, 668, 245

Gilbert, K. M., Guhathakurta, P., Kollipara, P., et al. 2009, ApJ, 705, 1275

Gültekin, K., Richstone, D. O., Gebhardt, K., et al. 2009, ApJ, 698, 198

Halliday, C., Davies, R. L., Kuntschner, H., et al. 2001, MNRAS, 326, 473

Hanes, D. A., Côté, P., Bridges, T. J., et al. 2001, ApJ, 559, 812

Harding, P., Morrison, H. L., Olszewski, E. W., et al. 2001, AJ, 122, 1397

Harris, W. E. 2009, ApJ, 703, 939

Haşegan, I. M. 2007, PhD thesis, Rutgers Univ.

Haşegan, M., Jordán, A., Côté, P., et al. 2005, ApJ, 627, 203

Helmi, A. 2004, ApJ, 610, L97

Helmi, A. 2008, A\&AR, 15, 145

Helmi, A., Navarro, J. F., Meza, A., Steinmetz, M., \& Eke, V. R. 2003, ApJ, 592, L25

Helmi, A., White, S. D. M., de Zeeuw, P. T., \& Zhao, H. 1999, Nature, 402, 53 Hernquist, L. 1987, ApJS, 64, 715

Hernquist, L. 1990, ApJ, 356, 359

Hernquist, L., \& Quinn, P. J. 1988, ApJ, 331, 682

Hernquist, L., \& Quinn, P. J. 1989, ApJ, 342, 1

Hernquist, L., \& Spergel, D. N. 1992, ApJ, 399, L117

Hibbard, J. E., \& Mihos, J. C. 1995, AJ, 110, 140

Hopkins, P. F., Bundy, K., Hernquist, L., Wuyts, S., \& Cox, T. J. 2010, MNRAS, 401, 1099

Huchra, J., \& Brodie, J. 1987, AJ, 93, 779

Ibata, R., Irwin, M., Lewis, G., Ferguson, A. M. N., \& Tanvir, N. 2001, Nature, 412, 49

Janowiecki, S., Mihos, J. C., Harding, P., et al. 2010, ApJ, 715, 972

Jílková, L., Jungwiert, B., Krízek, M., et al. 2010, in ASP Conf. Ser. 423, Galaxy Wars: Stellar Populations and Star Formation in Interacting Galaxies, ed. B. Smith, N. Bastian, S. J. U. Higdon, \& J. L. Higdon (San Francisco, CA: ASP), 243

Johnston, K. V. 1998, ApJ, 495, 297

Johnston, K. V., Bullock, J. S., Sharma, S., et al. 2008, ApJ, 689, 936

Johnston, K. V., Spergel, D. N., \& Haydn, C. 2002, ApJ, 570, 656

Jordán, A., McLaughlin, D. E., Côté, P., et al. 2007, ApJS, 171, 101

Khochfar, S., \& Silk, J. 2006, ApJ, 648, L21

Koch, A., Rich, R. M., Reitzel, D. B., et al. 2008, ApJ, 689, 958
Koposov, S. E., Rix, H.-W., \& Hogg, D. W. 2010, ApJ, 712, 260

Kormendy, J., Bender, R., Magorrian, J., et al. 1997, ApJ, 482, L139

Kormendy, J., Fisher, D. B., Cornell, M. E., \& Bender, R. 2009, ApJS, 182, 216

Krick, J. E., Bridge, C., Desai, V., et al. 2011, ApJ, 735, 76

Larsen, S. S., Brodie, J. P., Huchra, J. P., Forbes, D. A., \& Grillmair, C. J. 2001, AJ, 121, 2974

Law, D. R., Majewski, S. R., \& Johnston, K. V. 2009, ApJ, 703, L67

Lee, Y.-W., Gim, H. B., \& Casetti-Dinescu, D. I. 2007, ApJ, 661, L49

Liu, F. S., Mao, S., Deng, Z. G., Xia, X. Y., \& Wen, Z. L. 2009, MNRAS, 396, 2003

Lotz, J. M., Miller, B. W., \& Ferguson, H. C. 2004, ApJ, 613, 262

Mackey, A. D., Huxor, A. P., Ferguson, A. M. N., et al. 2010, ApJ, 717, L11

Martínez-Delgado, D., Gabany, R. J., Crawford, K., et al. 2010, AJ, 140, 962

McIntosh, D. H., Guo, Y., Hertzberg, J., et al. 2008, MNRAS, 388, 1537

McLaughlin, D. E. 1999, ApJ, 512, L9

McNeil, E. K., Arnaboldi, M., Freeman, K. C., et al. 2010, A\&A, 518, A44

Merrett, H. R., Kuijken, K., Merrifield, M. R., et al. 2003, MNRAS, 346, L62

Merrifield, M. R., \& Kuijken, K. 1998, MNRAS, 297, 1292

Mihos, J. C., Harding, P., Feldmeier, J., \& Morrison, H. 2005, ApJ, 631, L41

Miyazaki, S., Komiyama, Y., Sekiguchi, M., et al. 2002, PASJ, 54, 833

Mouhcine, M., Ibata, R., \& Rejkuba, M. 2011, MNRAS, 415, 993

Murphy, J. D., Gebhardt, K., \& Adams, J. J. 2011, ApJ, 729, 129

Naab, T., Johansson, P. H., \& Ostriker, J. P. 2009, ApJ, 699, L178

Navarro, J. F., Frenk, C. S., \& White, S. D. M. 1996, ApJ, 462, 563

Nolthenius, R., \& Ford, H. 1986, ApJ, 305, 600

Oser, L., Naab, T., Ostriker, J. P., \& Johansson, P. H. 2012, ApJ, 744, 63

Oser, L., Ostriker, J. P., Naab, T., Johansson, P. H., \& Burkert, A. 2010, ApJ, 725, 2312

Paturel, G., Petit, C., Prugniel, P., et al. 2003, A\&A, 412, 45

Peek, J. E. G., \& Graves, G. J. 2010, ApJ, 719, 415

Peñarrubia, J., Belokurov, V., Evans, N. W., et al. 2010, MNRAS, 408, L26

Peng, E. W., Jordán, A., Côté, P., et al. 2006, ApJ, 639, 95

Peng, E. W., Jordán, A., Côté, P., et al. 2008, ApJ, 681, 197

Perrett, K. M., Stiff, D. A., Hanes, D. A., \& Bridges, T. J. 2003, ApJ, 589, 790

Proctor, R. N., Forbes, D. A., Romanowsky, A. J., et al. 2009, MNRAS, 398, 91 Prugniel, P., Zeilinger, W., Koleva, M., \& de Rijcke, S. 2011, A\&A, 528, A128 Quinn, P. J. 1984, ApJ, 279, 596

Richtler, T., Salinas, R., Misgeld, I., et al. 2011, A\&A, 531, A119

Rix, H.-W., de Zeeuw, P. T., Cretton, N., van der Marel, R. P., \& Carollo, C. M. 1997, ApJ, 488, 702

Romanowsky, A. J., \& Kochanek, C. S. 2001, ApJ, 553, 722

Romanowsky, A. J., Strader, J., Spitler, L. R., et al. 2009, AJ, 137, 4956

Rudick, C. S., Mihos, J. C., Frey, L. H., \& McBride, C. K. 2009, ApJ, 699, 1518

Rudick, C. S., Mihos, J. C., Harding, P., et al. 2010, ApJ, 720, 569

Ruszkowski, M., \& Springel, V. 2009, ApJ, 696, 1094

Schuberth, Y., Richtler, T., Hilker, M., et al. 2010, A\&A, 513, A52

Searle, L., \& Zinn, R. 1978, ApJ, 225, 357

Shih, H.-Y., \& Méndez, R. H. 2010, ApJ, 725, L97

Spolaor, M., Hau, G. K. T., Forbes, D. A., \& Couch, W. J. 2010, MNRAS, 408, 254

Starkenburg, E., Helmi, A., Morrison, H. L., et al. 2009, ApJ, 698, 567

Stott, J. P., Collins, C. A., Burke, C., Hamilton-Morris, V., \& Smith, G. P. 2011, MNRAS, 414, 445

Strader, J., Brodie, J. P., \& Forbes, D. A. 2004, AJ, 127, 3431

Strader, J., Romanowsky, A. J., Brodie, J. P., et al. 2011, ApJS, 197, 33 (S+11)

Tal, T., van Dokkum, P. G., Nelan, J., \& Bezanson, R. 2009, AJ, 138, 1417

Toloba, E., Boselli, A., Cenarro, A. J., et al. 2011, A\&A, 526, A114

Valentinuzzi, T., Poggianti, B. M., Saglia, R. P., et al. 2010, ApJ, 721, L19

van der Wel, A., Holden, B. P., Zirm, A. W., et al. 2008, ApJ, 688, 48

van Dokkum, P. G., Whitaker, K. E., Brammer, G., et al. 2010, ApJ, 709, 1018

Varghese, A., Ibata, R., \& Lewis, G. F. 2011, MNRAS, 417, 198

Vazdekis, A., Cenarro, A. J., Gorgas, J., Cardiel, N., \& Peletier, R. F. 2003, MNRAS, 340, 1317

Weil, M. L., Bland-Hawthorn, J., \& Malin, D. F. 1997, ApJ, 490, 664

Woodley, K. A., \& Harris, W. E. 2011, AJ, 141, 27

Whiley, I. M., Aragón-Salamanca, A., De Lucia, G., et al. 2008, MNRAS, 387, 1253

Xue, X.-X., Rix, H.-W., Yanny, B., et al. 2011, ApJ, 738, 79

Zemp, M., Diemand, J., Kuhlen, M., et al. 2009, MNRAS, 394, 641 